\title{
Parallel Split-Join Networks for Shared Account Cross-domain Sequential Recommendations
}

\begin{abstract}
Wenchao Sun, Muyang Ma, Pengjie Ren*, Yujie Lin, Zhumin Chen, Zhaochun Ren, Jun Ma, Maarten de Rijke ${ }^{\mathrm{C}}$
Sequential recommendation is a task in which one models and uses sequential information about user behavior for recommendation purposes. We study sequential recommendation in a particularly challenging context, in which multiple individual users share a single account (i.e., they have a shared account) and in which user behavior is available in multiple domains (i.e., recommendations are cross-domain). These two characteristics bring new challenges on top of those of the traditional sequential recommendation task. First, we need to identify the behavior associated with different users and different user roles under the same account in order to recommend the right item to the right user role at the right time. Second, we need to identify behavior in one domain that might
\end{abstract} be helpful to improve recommendations in other domains.

In this work, we study shared account cross-domain sequential recommendation and propose Parallel Split-Join Network (PSJNet), a parallel modeling network to address the two challenges above. We use "split" to address the challenge raised by shared accounts; DSJNet learns role-specific representations and uses a gating mechanism to filter out, from mixed user behavior, information of user roles that might be useful for another domain. In addition, "join" is used to address the challenge raised by the cross-domain esetting; PSJNet learns cross-domain representations by combining the information from "split" and then transforms it to another Aomain. We present two variants of PSJNet, PSJNet-I and PSJNet-II. PSJNet-I is a "split-by-join" framework that splits the inixed representations to get role-specific representations and joins them to obtain cross-domain representations at each timestamp simultaneously. PSJNet-II is a "split-and-join" framework that first splits role-specific representations at each timestamp, and then cthe representations from all timestamps and all roles are joined to obtain cross-domain representations. We concatenate the inulomain and cross-domain representations to compute a recommendation score for each item. Both PSJNet-I and PSJNet-II can simultaneously generate recommendations for two domains where user behavior in two domains is synchronously shared at each timestamp.

We use two datasets to assess the effectiveness of PSJNet. The first dataset is a simulated shared account cross-domain sequential recommendation dataset obtained by randomly merging the Amazon logs from different users in the movie and book domains. The crecond dataset is a real-world shared account cross-domain sequential recommendation dataset built from smart TV watching logs of a Commercial organization. Our experimental results demonstrate that PSJNet outperforms state-of-the-art sequential recommendation Daselines in terms of MRR and Recall.

Index Terms-Parallel modeling, Shared account recommendation, Cross-domain recommendation, Sequential recommendation

\section{INTRODUCTION}

It is hard to apply traditional recommendation methods such , e.g., collaborative filtering (CF)-based methods [64] or natrix factorization (MF)-based models [40, 79], in recommendation scenarios in which user profiles may be absent. This jhappens when users are not logged in or the recommender - system does not track user-ids. For this reason, sequential Kecommendation (SR) has been proposed for session-based 'Trecommendations [30]. Compared with traditional recommendations, SR has natural advantages when it comes to sequential dynamics [27], i.e., SR methods may generate different lists of recommended items at different timestamps. The goal of $\mathrm{SR}$ is to generate recommendations based on a sequence of user behavior (e.g., a sequence of songs listened to, videos watched, or products clicked), where interactions are usually grouped by same time frame [46, 44, 12]. SRs have a wide

${ }^{*}$ Corresponding author.

This paper is a substantially extended version of [52]. The additions are three-fold. First, we unify the parallel modeling framework introduced in [52] into the PSJNet architecture introduced in this paper and propose a new model PSJNet-II that improves the performance over previous proposals $(\pi$-Net in [52] corresponds to PSJNet-I in this paper). Second, we build a new dataset for shared account cross-domain sequential recommendation by simulating shared account characteristics on a public dataset. Third, we carry out more experiments to test PSJNet-I and PSJNet-II. More than half of the experiments reported in this paper were not in [52] and all relevant result tables and figures are either new additions to the article or report new results. range of applications in many domains such as e-commerce, job websites, music and video recommendations [65]. And users usually have specific goals during the process, such as finding a good restaurant in a city, or listening to a song of a certain style or mood [59].

Early studies into SR are mostly based on Markov chains (MCs) [89] or Markov decision processes (MDPs) [65]. Sequences of items are considered as states and a state-transition matrix or function is learned to generate recommendations. In this way, the dynamic characteristics of SR are taken into account. However, because the states in a MC- or MDP-based method correspond to sequences of items, the state-transition matrix or function quickly becomes unmanageable in realistic scenarios [60]. Recurrent neural networks have demonstrated their effectiveness in sequence modeling in the field of natural language processing. Motivated by this, recent studies have introduced recurrent neural networks (RNNs) into SR [30] and today RNN-based models have been widely adopted in the context of SR. Various RNN architectures have been proposed to enhance SR, e.g., to make SRs context-aware [47], personalized [59], or deal with repeat behavior [61]. However, so far RNN-based methods focus on a single domain and none simultaneously considers the shared account and cross-domain scenarios.

In this paper, we study SR in a particularly challenging 
context, shared account cross-domain sequential recommendation (SAC-SR). In this context multiple individual users share a single account (i.e., they have a shared account) and user behavior is recorded in multiple domains (i.e., recommendations should be cross-domain). The shared account task is considered because in some recommendation applications people tend to share a single account, resulting in multiple "user roles" under each account. For example, in the smart TV recommendation scenario depicted in Figure 1, members of a family correspond to different user roles, e.g., "parents", "children", and they share a single account to watch videos. The existence of shared accounts makes it harder to generate relevant recommendations, because the behavior of multiple user roles is mixed together. Note that user roles do not explicitly represent specific users. We consider user roles instead of users because the number of user roles is smaller than that of users, and it is generally easier to distinguish different user roles than users. We consider the cross-domain task because it is a common phenomenon in practice: users use different platforms to access domain-specific services in daily life. We can get user behavior data from different domains during the same time period. For example, many smart TV platforms use different channels to provide different services, e.g., a video channel (domain) that offers movies or TV series and an educational channel that offers educational material, as depicted in Figure 1 User behavior in one domain may be helpful for improving recommendations in another domain [76, 66, 33] because user behavior in different domains may reflect similar user interests. For example, as illustrated in Figure 11, videos like "Mickey Mouse" in the video domain might help to predict the next item "School House Fun" in the educational domain because they reflect the same interest in the Disney cartoon character "Mickey Mouse" presumably by a child in this family. Although leveraging user behavior information from another domain may provide useful information to help improve the recommendation performance, this type of transfer is non-trivial because the behavior of multiple user roles is mixed and this may introduce noise. This raises an interesting challenge, namely how to identify behavior from one domain that might be helpful to improve recommendations in other domains while minimizing the impact of noisy information?

In prior work that focuses on shared accounts, a common approach is to capture user interests by extracting latent features from high-dimensional spaces that describe the relationships among user roles under the same account [73, 2, 86]. And in prior work on the cross-domain task, one common solution is to aggregate information from two domains 19. 28. 32], while another is to transfer knowledge from the source domain to the target domain [14, 88]. None of these methods can be directly applied to SAC-SR: either important sequential characteristics of SR are largely ignored or they rely on explicit user ratings, which are usually unavailable in SR. In our previous work [52], we have introduced an architecture ( $\pi$-Net) that addresses the above issues by simultaneously generating recommendations for two domains where user behavior from two domains is synchronously shared at each timestamp.
In this work, we generalize over the $\pi$-Net architecture with a more general framework, namely the Parallel SplitJoin Network (Parallel Split-Join Network (PSJNet)), that introduces the "split" and "join" concepts to address the shared account and cross-domain characteristics in SAC-SR. To address shared accounts, "split" is used to identify behavior of different user roles, where we employ a gating mechanism to extract role-specific representations containing information of some user roles that might be useful for another domain from mixed user behavior. To address the cross-domain aspect, "join" is used to discriminate and combine useful user behavior; we learn cross-domain representations by combining the information from "split" and then adopting it to another domain.

Specifically, PSJNet is organized in four main modules, namely a sequence encoder, a split unit, a join unit, and a hybrid recommendation decoder. The sequence encoder module encodes the current sequence of mixed user behavior from each domain into a sequence of in-domain representations. Then, depending on how "split" and "join" are implemented, we present two PSJNet variants, i.e., PSJNet-I and PSJNetII. PSJNet-I (which corresponds to $\pi$-Net) employs a "Splitby-Join" scheme where it splits the mixed representations to get role-specific representations and joins them to get cross-domain representations at each timestamp simultaneously. PSJNet-II employs a "Split-and-Join" scheme where it first splits role-specific representations at each timestamp, and then the representations from all timestamps and all roles are joined to obtain cross-domain representations. For both variants, "split" and "join" are operated in a parallel recurrent way, which means that they can synchronously share information across both domains at each timestamp. Finally, the hybrid recommendation decoder module estimates the recommendation scores for each item based on the information from both domains, i.e., the in-domain representations from the target domain and the cross-domain representations from the complementary domain. During learning, PSJNet is jointly trained on two domains in an end-to-end fashion.

To assess the effectiveness of PSJNet, we need datasets that exhibit both shared account and cross-domain characteristics. To the best of our knowledge, there is no such real-world dataset that is publicly available. We construct two datasets for SAC-SR. The first dataset is a simulated SAC-SR dataset obtained by randomly merging the logs from different users in the movie and book domains from a well-known Amazon dataset 1 Although the dataset can satisfy experimental requirements, the merged user behavior is not realistic and is unlikely to reflect realistic scenarios. Therefore, we build a second dataset from smart TV watching logs of a commercial company, which is a real-world SAC-SR scenario. We release both datasets to facilitate future research. We carry out extensive experiments on both datasets. The experimental results show that PSJNet outperforms state-of-the-art baselines in terms of MRR and Recall. We also conduct ablation studies to show that the proposed parallel "split" and "join" schemes are effective and useful for SAC-SR.

${ }^{1}$ http://jmcauley.ucsd.edu/data/amazon/ 


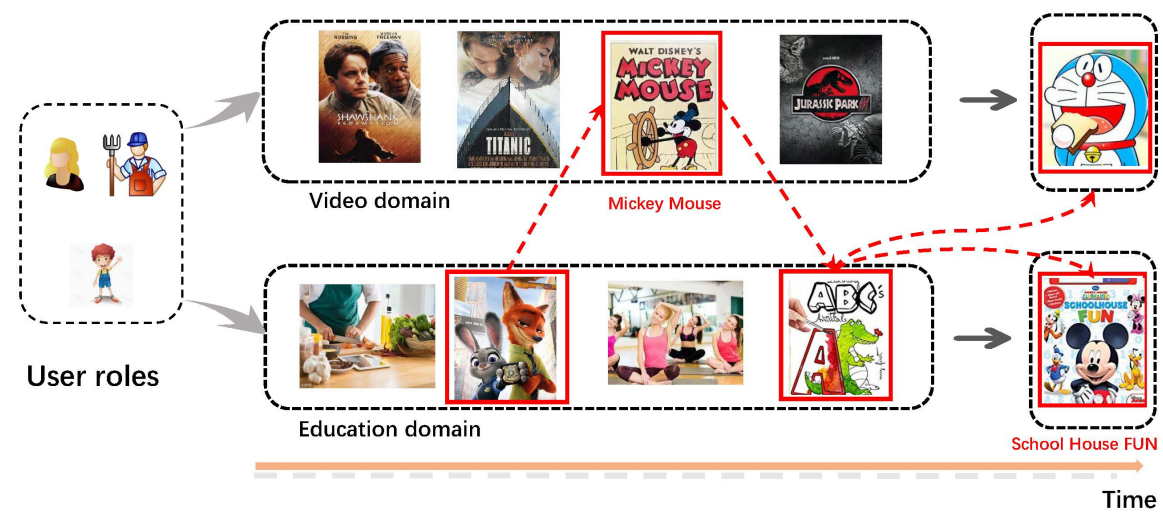

Fig. 1: The smart TV scenario provides a natural example of shared account cross-domain sequential recommendation (SAC-SR). Here, members of a family (the "user roles") share the same account. Moreover, the video domain contains various movies, TV series, cartoons, etc. The education domain contains educational programs and technical tutorials, etc. Boxed items reflect similar user interests. Red lines show the interactions and connections between user behavior in the two domains.

The additional contributions of this paper compared to our previous work in [52] are as follows:

- We present the PSJNet framework, which introduces the "split" and "join" concepts to address the shared account and cross-domain characteristics of SAC-SR.

- We reformulate the previous proposal $\pi$-Net as PSJNet-I within the PSJNet framework, and propose a new variant PSJNet-II that further improves the performance.

- We carry out experiments on two datasets for SAC-SR. One is constructed by simulating shared account characteristics on a public dataset, the other is a real-world dataset. And we conduct additional experiments on these two datasets to show the effectiveness of the two PSJNet variants.

\section{RELATED WORK}

We consider three types of related work: sequential recommendations, shared account recommendations, and crossdomain recommendations.

\section{A. Sequential recommendations}

It is hard to capture sequential dynamics in recommendation scenarios with classical recommendation methods such as matrix factorization (MF)- or collaborative filtering (CF)-based methods. Instead, dedicated methods have been developed for SR or next basket recommendation.

\section{1) Traditional methods}

The traditional approaches for SR are mostly based on Markov chains (MCs) [89] or Markov decision processes (MDPs) [65] to predict a user's next action given their last action [75]. Zimdars et al. [89] are the first to propose MCs for web page recommendation. They investigate how to extract sequential patterns to learn the next state using probabilistic decision-tree models. To further improve the performance, Shani et al. [65] propose an MDP-based recommendation method, where the next recommendation can be computed through the transition probabilities among items. To combine the advantages of MF and MC-based methods, Rendle et al. [63] propose a method based on personalized transition graphs over an underlying MC. They show that the proposed method subsumes both a common MC and the normal MF model. Yap et al. [82] introduce a competence score measure in personalized sequential pattern mining for next-item recommendations. Chen et al. [10] take playlists as an MC, and propose logistic Markov embeddings to learn representations of songs for playlists prediction. Wu et al. [78] propose Personalized Markov Embedding (PME) to consider sequential singing behavior for the next song recommendation. They embed users and songs into a Euclidean space, where the distance between songs and users represent their relationships. Given a user's last song, they can generate personalized recommendations by ranking the candidate songs according to the relationships.

Lu et al. [50] argue that source domain data is not always consistent with the observations in the target domain, which may misguide the target domain recommendation. They propose a criterion based on empirical prediction error and its variance to better capture the consistency across domains in CF settings. To address the sparsity and long-tailed distribution issues of most recommendation datasets and take sequential dynamics into consideration at the same time, $\mathrm{He}$ and McAuley [27] propose to combine the advantages of MCbased methods and CF-based methods. They fuse a similaritybased method with MC to learn a personalized weighting scheme over the sequence of items to characterize users in terms of both interests and the strength of sequential behavior.

All of the MC or MDP-based sequential recommendation methods mentioned above show improvements by modeling sequential dynamics. However, a major issue they share is that they can only consider a very short sequence (e.g., the most recent five items in [65]), because the state space quickly becomes unmanageable when taking long sequences into account [60].

\section{2) Deep learning-based methods}

Recently, recurrent neural networks (RNNs) have been devised to model variable-length sequential data [83, 20]. Hidasi et al. [30] are the first to apply RNNs to sequential recommendation and achieve significant improvements over traditional methods. They utilize session-parallel mini- 
batch training and employ ranking-based loss functions to train the recommendation model. In later work, they propose data augmentation techniques to improve the performance of RNNs [69].

Contextual information has proved to be very important for behavior modeling in traditional recommendations. Liu et al. [47] incorporate contextual information into SR and propose a context-aware RNN model. Instead of using the constant input matrix and transition matrix from conventional RNN models, their CA-RNN employs adaptive matrices. The authors use context-specific input matrices to capture external conditions under which user behavior happens, such as time, location, weather and so on. They also use context-specific transition matrices to capture how the length of time intervals between adjacent behavior in historical sequences affects the transition of global sequential features. Hidasi et al. [31] investigate how to add item property information such as text and images to an RNN framework and introduce a number of parallel RNN architectures (p-RNNs); they propose alternative training strategies for $\mathrm{p}$-RNNs that suit them better than standard training. Bogina and Kuflik [6] explore user's dwell time based on an existing RNN-based framework by boosting items above a predefined dwell time threshold. Cui et al. [15] incorporate visual and textual information and propose MVRNN to alleviate the cold start problem.

Donkers et al. [18] introduce a new gated architecture with additional input layers for gated recurrent units (GRUs) to explicitly represent individual users, for the purpose of generating personalized next item recommendations. Chen et al. [8] propose a dictionary learning-based approach to model a user's static and dynamic preferences. They use a GRU to translate a user's sequential behavior into dynamic user preferences. Tan et al. [68] propose a dynamic memorybased recurrent attention network for modeling long behavior sequences. Quadrana et al. [59] propose a hierarchical RNN model that can be used to generate personalized sequential recommendations. Li et al. [44] explore a hybrid encoder with an attention mechanism to model the user's sequential behavior and intent to capture the user's main purpose in the current sequence. Zhuang et al. [88] propose a novelty seeking model based on sequences in multi-domains to model an individual's propensity by transferring novelty seeking traits learned from a source domain for improving the accuracy of recommendations in the target domain. Tang and Wang [70] propose a convolutional sequence embedding recommendation model for personalized top-n sequential recommendation to address the more recent items where they argue that more recent items in a sequence have a larger impact on the next item. Ren et al. [61] propose a repeat-aware RNN model to address the repeat consumption in SR, which is a common phenomenon in many recommendation scenarios where the same item is repeatedly re-consumed. They incorporate a new repeat recommendation mechanism into RNNs that can choose items from a user's history and recommends them at the right time.

Memory enhanced RNNs have been well studied for SR recently. Chen et al. [11] argue that existing SR methods usually embed a user's historical records into a single latent representation, which may have lost the per item- or featurelevel correlations between a user's historical records and future interests. They introduce a memory mechanism to SR and design a memory-augmented neural network integrated with insights from collaborative filtering.

Huang et al. [34] propose a knowledge enhanced SR model to capture fine-grained user interests from interaction sequences. They integrate RNN-based networks with a key-value memory network. They further incorporate knowledge base information to enhance the learned semantic representations. Ma et al. [53] propose a cross-attention memory network to perform the mention recommendation task for multi-modal tweets where they make full use of both textual and visual information. Huang et al. [35] introduce a taxonomy-aware multi-hop reasoning network, which integrates a basic GRUbased sequential recommender with an elaborately designed memory-based multi-hop reasoning architecture. They incorporate taxonomy data as structural knowledge to enhance the reasoning capacity.

Wang et al. [74] hypothesize that the collaborative information contained in neighborhood sequences (that have been generated previously by other users and reflect similar user intents as the current sequence) might help to improve recommendation performance for the current sequence. They propose a RNN model with two parallel memory modules: one to model a user's own information in the current sequence and the other to exploit collaborative information in neighborhood sequences [37]. Most recently, Li et al. [43] propose a transformer-based structured intent-aware model that first extracts intents from sequential contexts, and then adopts an intent graph to capture the correlations among user intents.

\section{B. Shared account recommendations}

Most recommender systems assume that every account in the data represents a single user. However, multiple users often share a single account. A typical example is a smart TV account for the whole family.

Previous approaches to shared account recommendations typically first identify users and then make personalized recommendations [73, 86, 14, 2]. Zhang et al. [84] are the first to study user identification, in which they use linear subspace clustering algorithms; they recommend the union of items that are most likely to be rated highly by each user. Bajaj and Shekhar [3] propose a similarity-based channel clustering method to group similar channels for IPTV accounts, and they use the Apriori algorithm to separate users that are merged under a single account. After that, they use personal profiles to recommend additional channels to the account. Wang et al. [77] assume that different users consume services in different periods. They decompose users based on mining different interests over different time periods from consumption logs. Finally, they use a standard User-KNN method to generate recommendations for each identified user. Yang et al. [81] also analyze the similarity of the proportion of each type of items within a time period to judge whether a sequence is generated by the same user. Then, they generate recommendations by recommending personalized genres to the identified users. 
Lesaege et al. [41] develop a time-aware user identification model based on Latent Dirichlet Allocation using a hidden variable to represent the user, and assume consumption times to be generated by latent time topics. Yang et al. [80] identify users by using a projection based unsupervised method, and then use Factorization Machine techniques to predict a user's interest based on historical information to generate personalized recommendations. Jiang et al. [38] propose an unsupervised learning-based framework to identify users and differentiate the interests of users and group sessions by users. They construct a heterogeneous graph to represent items and use a normalized random walk to create item-based session embeddings. A hybrid recommender is then proposed that linearly combines the account-level and user-level item recommendation by employing Bayesian personalized ranking matrix factorization [62].

\section{Cross-domain recommendations}

Cross-domain recommendation concerns data from multiple domains, which has proven to be helpful for alleviating the cold start problem [1, 5] and data sparsity issues [42, 57]. There is an assumption that there exists overlap in information between users and/or items across different domains [19, 21].

\section{1) Traditional methods}

There are two main ways for dealing with cross-domain recommendations [22]. One is to aggregate knowledge between two domains. Berkovsky et al. [4] propose four mediation techniques to solve the data sparsity problem by merging user interests and extracting common attributes of users and items. Tang et al. [71] propose a cross-domain topic learning model to address three challenges in cross-domain collaboration recommendation: sparse connections (cross-domain collaborations are rare); complementary expertise (cross-domain collaborators often have different expertise and interest) and topic skewness (cross-domain collaboration topics are focused on a subset of topics) Shapira et al. [66] compare several collaborative methods to demonstrate the effectiveness of utilizing available preference data from Facebook. Loni et al. [49] model user interests by using MF separately on different domains, and then incorporate user interaction patterns that are specific to particular types of items to generate recommendations on the target domain. Do et al. [16] propose to discover both explicit and implicit similarities from latent factors across domains based on matrix tri-factorization. Zhuang et al. [87] propose a consensus regularization classifier framework by considering both local data available in source domain and the prediction consensus with the classifiers learned from other source domains. Cao et al. [7] construct a nonparametric Bayesian framework by jointly considering multiple heterogeneous link prediction tasks between users and different types of items. Chen et al. [9] exploit the users and items shared between domains as a bridge to link different domains by embedding all users and items into a low-dimensional latent space between different domains. Liu et al. [48] utilize both MF and an attention mechanism for fine-grained modeling of user preferences; the overlapping cross-domain user features are combined through feature fusion.
The other approach to cross-domain recommendation is to transfer knowledge from the source domain to the target domain. Hu et al. [33] propose tensor-based factorization to share latent features between different domains. Cremonesi and Quadrana [14] propose a code-book-transfer to transfer rating patterns between domains. Kanagawa et al. [39] propose a content-based approach to learn semantic information between domains. Doan and Sahebi [17] propose a transitionbased cross-domain collaborative filtering method to capture both within- and between-domain transitions of user feedback sequences. Zhang et al. [85] propose a method that not only transfers item's learned latent factors, but also selectively transfers user's latent factors.

\section{2) Deep learning-based methods}

Deep learning is well suited to transfer learning as it can learn high-level abstractions among different domains [56]. Lian et al. [45] introduce a factorization framework to tie collaborative filtering and content-based filtering together; they use neural networks to build user and item embeddings. Elkahky et al. [19] propose a multi-view deep learning recommendation system by using rich auxiliary features to represent users from different domains based on deep structured semantic model (DSSM) [36]. Fernández-Tobías et al. [23] address the cold-start issue in a target domain by exploiting user interests from a related auxiliary domain. They show that cross-domain information is useful to provide more accurate and diverse recommendations when user feedback in the target domain is scarce or not available at all. Hu et al. [32] propose a model using a cross-stitch network [55] to learn complex useritem interaction relationships based on neural collaborative filtering [28]. Zhuang et al. [88] propose a novelty-seeking model to fully characterize users' novelty-seeking trait so as to obtain a better performance across domains. Wang et al. [76] are the first to introduce the problem of cross-domain social recommendations; they combine user-item interactions in information domains (such as online travel planning) and user-user connections in social network services (such as Facebook or Twitter) to recommend relevant items of information domains to target users of social domains; user and item attributes are leveraged to strengthen the embedding learning.

Although the methods proposed in the studies listed above have been proven to be effective in many applications, they either cannot be applied to sequential recommendations or do not consider the shared account or cross-domain characteristics. In our previous work, we have proposed $\pi$-Net in order to address shared account and cross-domain characteristics in sequential recommendations by extracting information of different user roles under the same account and transferring it to a complementary domain at each timestamp [52]. In this work, we present a more general framework called PSJNet: $\pi$-Net can be viewed as a particular instantiation of PSJNet and we propose another instantiation that further improves the recommendation performance over $\pi$-Net.

\section{METHOD}

In this section, we first provide a formulation of the shared account cross-domain sequential recommendation (SAC-SR) 
problem. Then, we introduce PSJNet and describe two instantiations of the framework. For each variant, we give a highlevel introduction and describe each component in detail.

\section{A. Shared account cross-domain sequential recommendation}

We represent a cross-domain behavior sequence (e.g., watching videos, reading books) from a shared account as $S=\left\langle A_{1}, B_{1}, B_{2}, \ldots, A_{i}, \ldots, B_{j}, \ldots\right\rangle$, where $A_{i} \in \mathbb{A}(1 \leq$ $i \leq N)$ is the index of a single consumed item in domain $A$; $\mathbb{A}$ is the set of all items in domain $A ; B_{j} \in \mathbb{B}(1 \leq j \leq M)$ is the index of a single consumed item in domain $B ; \mathbb{B}$ is the set of all items in domain $B ; N$ and $M$ are the number of items in the sequences from domain $A$ and $B$, respectively. Given $S$, SAC-SR tries to predict the next item by computing the recommendation probabilities for all candidates in two domains respectively, as shown in Eq. 1 .

$$
\begin{aligned}
P\left(A_{i+1} \mid S\right) & \sim f_{A}(S) \\
P\left(B_{j+1} \mid S\right) & \sim f_{B}(S),
\end{aligned}
$$

where $P\left(A_{i+1} \mid S\right)$ denotes the probability of recommending the item $A_{i+1}$ that will be consumed next in domain $A$. Also, $f_{A}(S)$ is the model or function used to estimate $P\left(A_{i+1} \mid S\right)$. Similar definitions apply to $P\left(B_{j+1} \mid S\right)$ and $f_{B}(S)$.

The main differences between SAC-SR and traditional SR are two-fold. First, in SAC-SR, $S$ is generated by multiple users (e.g., family members) while it is usually generated by a single user in SR. Second, SAC-SR considers information from both domains for the particular recommendations in one domain, i.e., $S$ is a mixture of behavior from multiple domains. In this paper, we only consider two domains but the ideas easily generalize to multiple domains.

Next, we will describe two PSJNet variants in detail. The key idea of PSJNet is to learn a recommendation model that can first extract the information of some specific user roles from domain $A$, and then transfer the information to domain $B$, and combine it with the original information from domain $B$ to improve recommendation performance for domain $B$, and vice versa. This process is achieved in a parallel way, which means that the information from both domains is shared recurrently.

\section{B. Sequence encoder}

Both variants of PSJNet that we consider use the same sequence encoder. Like previous studies [30, 69, 59], we use a RNN to encode a sequence $S$. Here, we employ two separate GRUs as the recurrent units to encode the items from domain $A$ and domain $B$ respectively. And the GRU is given as follows:

$$
\begin{aligned}
z_{t} & =\sigma\left(W_{z}\left[\operatorname{emb}\left(x_{t}\right), h_{t-1}\right]\right) \\
r_{t} & =\sigma\left(W_{r}\left[\operatorname{emb}\left(x_{t}\right), h_{t-1}\right]\right) \\
\widetilde{h_{t}} & =\tanh \left(W_{\widetilde{h}}\left[\operatorname{emb}\left(x_{t}\right), r_{t} \odot h_{t-1}\right]\right) \\
h_{t} & =\left(1-z_{t}\right) \odot h_{t-1}+z_{t} \odot \widetilde{h_{t}},
\end{aligned}
$$

where $W_{z}, W_{r}$, and $W_{\widetilde{h}}$ are weight matrices; $\operatorname{emb}\left(x_{t}\right)$ is the item embedding of item $x$ at timestamp $t$; and $\sigma$ denotes the sigmoid function. The initial state of the GRUs is set to zero vectors, i.e., $h_{0}=0$. Through the sequence encoder we obtain $H_{A}=\left\langle h_{A_{1}}, h_{A_{2}}, \ldots, h_{A_{i}}, \ldots, h_{A_{N}}\right\rangle$ for domain $A$, and $H_{B}=\left\langle h_{B_{1}}, h_{B_{2}}, \ldots, h_{B_{j}}, \ldots, h_{B_{M}}\right\rangle$ for domain $B$. We consider the last state as the in-domain representation, i.e., $h_{A}=h_{A_{N}}$ for domain $A$ and $h_{B}=h_{B_{M}}$ for domain $B$. The in-domain representations are combined with the crossdomain representations (i.e., $h_{(A \rightarrow B)}$ or $h_{(B \rightarrow A)}$ ) to compute the final recommendation score. In the next two subsections, we will describe two PSJNet instantiations that adopt different frameworks to learn the cross-domain representations.

\section{PSJNet-I}

In this subsection, we describe PSJNet-I in detail. PSJNet-I is a reformulation of $\pi$-Net [52] within the PSJNet framework, where we reformulate the shared account filter unit (SFU) and the cross-domain transfer unit (CTU) as a split-by-join unit. Figure 2 provides an overview of PSJNet-I. PSJNet-I is a "Split-by-Join" framework where it gets the role-specific representations from the mixed user behavior and simultaneously joins them at each timestamp. Then the representations are transformed to another domain as cross-domain representations. PSJNet-I consists of three main components: (1) a sequence encoder (see Section III-B), (2) a split-by-join unit, and (3) a hybrid recommendation decoder (see Section III-E). The sequence encoder encodes the behavior sequences of each domain into high-dimensional hidden representations. The split-by-join unit takes each domain's representations as input and tries to first split the representations of specific user roles, and then joins and transforms them to another domain at each timestamp $t$. The matching decoder combines the information from both domains and generates a list of recommended items. Please refer to Sections III-B and III-E for details of the sequence encoder and the hybrid recommendation decoder, respectively. In this subsection, we focus on the core module (i.e., the split-by-join unit) of PSJNet-I.

\section{1) Split-by-join unit}

Under the shared account scenario, the behavior recorded under the same account is generated by different user roles. In practice, not all user roles that share the account are active in all domains. Besides, even though some user roles are active in the same domain, they may have different interests. For example, in the smart TV scenario, children may occupy the majority of the educational channel, while adults dominate the video TV channel.

The outputs $H_{A}$ or $H_{B}$ of the sequence encoder are the mixed representations of all user roles sharing the same account. To learn role-specific representations from these mixed representations, we propose a split-by-join unit, as shown in Figure 3 The split-by-join unit can be applied bidirectionally from "domain $A$ to domain $B$ " and "domain $B$ to domain $A$," meaning that the information is extracted from one domain and transferred to the other domain. Here, we take the "domain $A$ to domain $B$ " direction and achieving recommendations in domain $B$ as an example. To learn role-specific representations, we need to know the number of user roles under each account, which is, unfortunately, unavailable in most cases. In this work, we assume that there are $K$ latent roles $\left(r_{1}\right.$, 


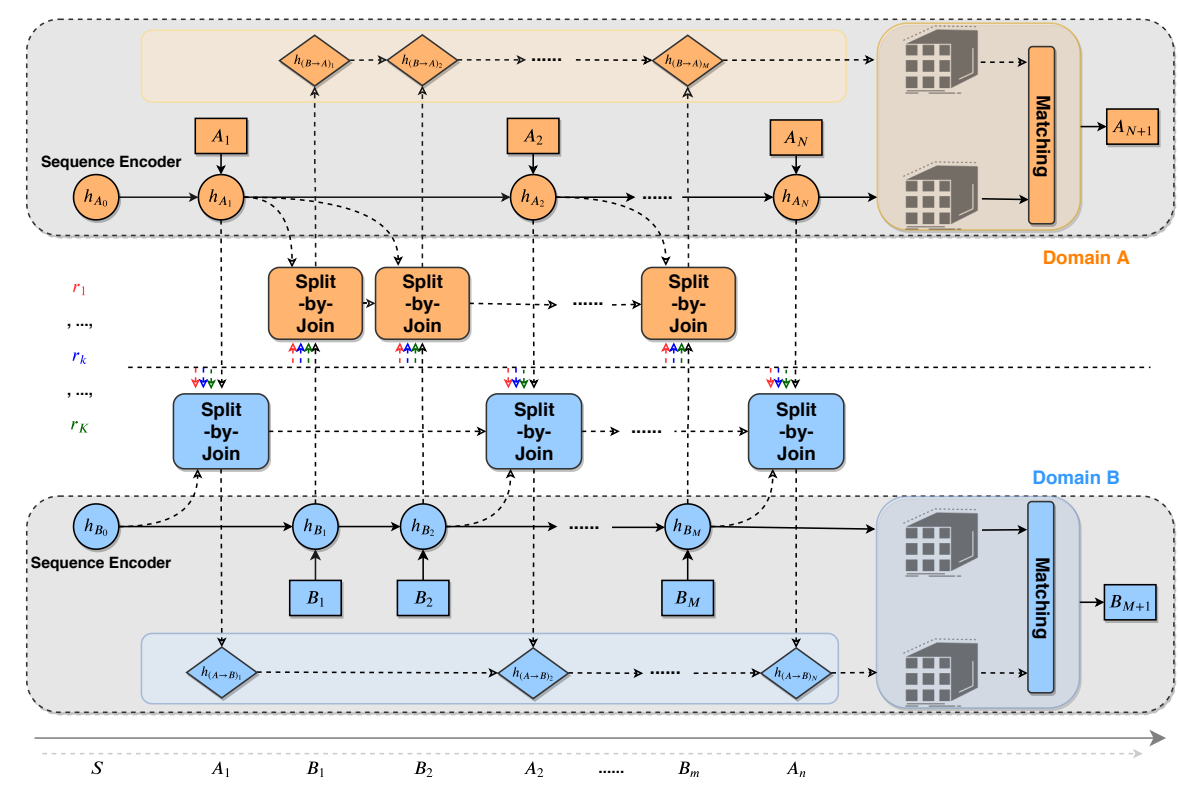

Fig. 2: An overview of PSJNet-I. The orange and blue colors represent different domains. Red, purple and green represent different user roles. Section $\mathrm{III-C}$ contains a walkthrough of the model.

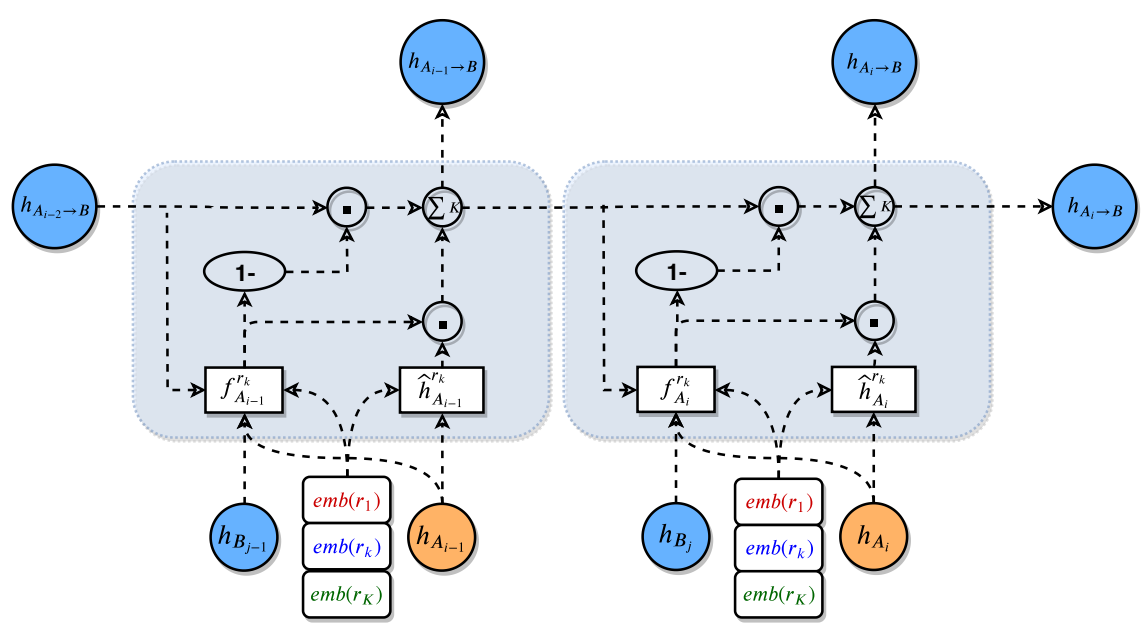

Fig. 3: The split-by-join unit illustrated while transforming information from domain $A$ to domain $B$.

$\left.r_{2}, \ldots, r_{k}, \ldots, r_{K}\right)$ under each account. For example, in a family account, the user roles may correspond to child, male parent, female parent, etc. We first embed each latent role into $\operatorname{emb}\left(r_{k}\right)(1 \leq k \leq K)$, which represents and encodes the latent interests of each role. Then, we split the specific representation for role $r_{k}$ at timestamp $i$ in domain $A$ with Eq. 3 .

$$
h_{A_{i}}^{r_{k}}=f_{A_{i}}^{r_{k}} \odot \widehat{h}_{A_{i}}^{r_{k}}+\left(1-f_{A_{i}}^{r_{k}}\right) \odot h_{A_{i-1} \rightarrow B},
$$

where $\odot$ denotes element-wise multiplication. Mathematically, the representation $h_{A_{i}}^{r_{k}}$ is a combination of two representations $\widehat{h}_{A_{i}}^{r_{k}}$ and $h_{A_{i-1} \rightarrow B}$ balanced by $f_{A_{i}}^{r_{k}}$. A higher value of $f_{A_{i}}^{r_{k}}$ means that item $A_{i}$ is more likely generated by $r_{k}$ and we should pay more attention to $r_{k}$ 's related representation $\widehat{h}_{A_{i}}^{r_{k}}$. A lower value of lower $f_{A_{i}}^{r_{k}}$ means that item $A_{i}$ might not be related to $r_{k}$ and we should inherit more information from previous time steps.
Next, we introduce the definitions of the three parts of Eq. 3 one by one.

(a) We propose a gating mechanism to implement $f_{A_{i}}^{r_{k}}$ in Eq. 4 .

$$
\begin{aligned}
f_{A_{i}}^{r_{k}}=\sigma\left(W_{f_{A}} \cdot h_{A_{i}}+\right. & W_{f_{B}} \cdot h_{B_{j}}+U_{f} \cdot h_{A_{i-1} \rightarrow B} \\
& \left.+V_{f} \cdot e m b\left(r_{k}\right)+b_{f}\right),
\end{aligned}
$$

where - means matrix multiplication; $W_{f_{A}}, W_{f_{B}}, U_{f}$ and $V_{f}$ are the parameters; $b_{f}$ is the bias term; $h_{A_{i}}$ and $h_{B_{j}}$ are the mixed representations of domain $A$ and $B$ at timestamp $i$ and $j$, respectively. Note that $B_{j}$ is the last item from domain $B$ before $A_{i}$ in the mixed sequence. $h_{A_{i-1} \rightarrow B}$ is the previous output, which will be explained later (under item (c) ). After the sigmoid function $\sigma$, each value of $f_{A_{i}}^{r_{k}}$ falls into $(0,1)$. Thus, the gating score $f_{A_{i}}^{r_{k}}$ controls the amount of information of $r_{k}$ to transfer from domain $A$ to domain $B$. It should be noted that each latent representation $e m b\left(r_{k}\right)$ indicates the distribution of user 
roles with similar interests under each account, and it does not explicitly represents a specific user.

(b) $\widehat{h}_{A_{i}}^{r_{k}}$ is the candidate representation for $r_{k}$ at timestamp $i$ in domain $A$, which is computed based on the mixed representation $h_{A_{i}}$, the filtered previous output $h_{A_{i-1} \rightarrow B}$, and the user role $r_{k}$ 's latent interest $\operatorname{emb}\left(r_{k}\right)$, as shown in Eq. 5 .

$$
\begin{gathered}
\widehat{h}_{A_{i}}^{r_{k}}=\tanh \left(W_{h} \cdot h_{A_{i}}+U_{h} \cdot h_{A_{i-1} \rightarrow B}+\right. \\
\left.V_{h} \cdot \operatorname{emb}\left(r_{k}\right)+b_{h}\right)
\end{gathered}
$$

where $W_{h}, U_{h}$ and $V_{h}$ are the parameters; $b_{h}$ is the bias term.

(c) $h_{A_{i} \rightarrow B}$ is the final output of the cross-domain representation at timestamp $i$ from domain $A$ to domain $B$, which is calculated by joining each role-specific representation $h_{A_{i}}^{r_{k}}$ :

$$
h_{A_{i} \rightarrow B}=\frac{1}{K} \sum_{k=1}^{K}\left(h_{A_{i}}^{r_{k}}\right) .
$$

Note that $h_{A_{i} \rightarrow B}$ is recurrently updated with Eq. 3 and 6

Using Eq. 3 and 6, we obtain a sequence of representations $\left\langle h_{A_{1} \rightarrow B}, \ldots, h_{A_{N} \rightarrow B}\right\rangle$. We need to combine and transfer $\left\langle h_{A_{1} \rightarrow B}, \ldots, h_{A_{N} \rightarrow B}\right\rangle$ to domain $B$. We achieve this by employing another GRU to recurrently encode $h_{A_{i} \rightarrow B}$ at each timestep to obtain $h_{(A \rightarrow B)_{i}}$, as shown in Eq. 7 ;

$$
h_{(A \rightarrow B)_{i}}=\operatorname{GRU}\left(h_{A_{i} \rightarrow B}, h_{(A \rightarrow B)_{i-1}}\right),
$$

where $h_{A_{i} \rightarrow B}$ is the representation filtered from domain $A ; h_{(A \rightarrow B)_{i-1}}$ is the previous transformed representation at timestamp $i-1$. The initial state is also set to zero vectors, i.e., $h_{(A \rightarrow B)_{0}}=0$. We set the cross-domain representation from domain $A$ to domain $B$ (i.e., $h_{(A \rightarrow B)}$ ) as the last timestamp representation $h_{(A \rightarrow B)_{N}}$, where $N$ is sequence length of domain $A$.

\section{PSJNet-II}

In this subsection, we describe PSJNet-II, our second solution for SAC-SR, in detail. Unlike PSJNet-I, PSJNet-II is a "Split-and-Join" framework, which means that it first splits role-specific representations from the mixed user behavior at each timestamp. Then the role-specific representations are transformed to another domain. Finally, it joins the rolespecific representations as cross-domain representations. Figure 4 provides an overview of PSJNet-II. PSJNet-II consists of four main components: (1) a sequence encoder (see Section III-B, (2) a split unit, (3) a join unit, and (4) a hybrid recommendation decoder (see Section III-E). PSJNetII uses the same sequence encoder and matching decoder architectures as PSJNet-I. Please refer to Section III-B and III-E for details of the sequence encoder and the hybrid recommendation decoder. In this subsection, we focus on the core modules (i.e., the split unit and join unit) of PSJNet-II.

\section{1) Split unit}

The split unit is shown in Figure 5 The differences with the split-by-join unit of PSJNet-I are marked in yellow. As with PSJNet-I, PSJNet-II also assumes that there are $K$ latent roles under each account. We split the specific representation for role $r_{k}$ at timestamp $i$ in domain $A$ with Eq. 8.

$$
h_{A_{i} \rightarrow B}^{r_{k}}=f_{A_{i}}^{r_{k}} \odot \widehat{h}_{A_{i}}^{r_{k}}+f_{A_{i}}^{\text {none }} \odot h_{A_{i-1} \rightarrow B}^{r_{k}},
$$

where $f_{A_{i}}^{\text {none }}$ is a special gate that handles the case when none of the information from $r_{k}$ at $i$ (i.e., $\widehat{h}_{A_{i}}^{r_{k}}$ ) is useful and we should inherit more information from previous time steps, see Eq. 9 .

$f_{A_{i}}^{\text {none }}=\sigma\left(W_{f_{A}} \cdot h_{A_{i}}+W_{f_{B}} \cdot h_{B_{j}}+U_{f} \cdot h_{A_{i-1} \rightarrow B}+b_{f}\right)$.

We add a normalization constraint to force the sum of $f_{A_{i}}^{r_{k}}$ and $f_{A_{i}}^{\text {none }}$ to 1 :

$$
f_{A_{i}}^{n o n e}+\sum_{k=1}^{K} f_{A_{i}}^{r_{k}}=1 .
$$

We use similar definitions of $f_{A_{i}}^{r_{k}}$ (Eq. 4) and $\widehat{h}_{A_{i}}^{r_{k}}$ (Eq. 5) as in PSJNet-I, except that $h_{A_{i-1} \rightarrow B}$ is replaced with $h_{A_{i-1} \rightarrow B}^{r_{k}}$. The differences from split-by-join unit are two-fold. First, $h_{A_{i} \rightarrow B}^{r_{k}}$ is not joined with respect to all roles. Second, instead of learning independent gates for different roles, we require that all gate values from all roles (and $f_{A_{i}}^{\text {none }}$ ) are summed to 1 .

After Eq. 8, we get a sequence of representations $\left\langle h_{A_{1} \rightarrow B}^{r_{k}}, \ldots, h_{A_{n} \rightarrow B}^{r_{k}}\right\rangle$ for each user role $r_{k}$. We combine and transfer $\left\langle h_{A_{1} \rightarrow B}^{r_{k}}, \ldots, h_{A_{n} \rightarrow B}^{r_{k}}\right\rangle$ to domain $B$ by employing another GRU, as shown in Eq. 11.

$$
h_{(A \rightarrow B)_{i}}^{r_{k}}=\operatorname{GRU}\left(h_{A_{i} \rightarrow B}^{r_{k}}, h_{(A \rightarrow B)_{i-1}}^{r_{k}}\right),
$$

where $h_{A_{i} \rightarrow B}^{r_{k}}$ is the representation filtered from domain $A$ for role $r_{k}$.

\section{2) Join unit}

The join unit is shown in Figure 6. After the split unit, we obtain $K$ sequences of transformed representations $\left\langle h_{(A \rightarrow B)_{1}}^{r_{k}}, \ldots, h_{(A \rightarrow B)_{N}}^{r_{k}}\right\rangle$ from domain $A$ to domain $B$. To join them, we first compute a similarity matrix $S^{I} \in \mathbb{R}^{M \times N}$ between the transformed representations and the in-domain representations $\left\langle h_{B_{1}}, \ldots, h_{B_{M}}\right\rangle$ from domain $B$. Each similarity $S_{(i, j)}^{I}$ is computed with Eq. 12

$$
S_{(i, j)}^{I}=v_{S}^{T} \cdot\left(W_{i} \cdot h_{(A \rightarrow B)_{i}}^{r_{k}}+W_{j} \cdot h_{B_{j}}\right),
$$

where $v_{S}^{T}, W_{i}$ and $W_{j}$ are parameters.

Then we pick the maximum similarity $S_{i}^{I}$ between each $h_{(A \rightarrow B)_{i}}^{r_{k}}$ and all $h_{B_{j}}$. $S_{i}^{I}$ signifies that $h_{(A \rightarrow B)_{i}}^{r_{k}}$ is more representative for role $r_{k}$ in domain $B$ because it has the closest similarity to a representation $h_{B_{j}}$ in domain $B$ :

$$
S_{i}^{I}=\max _{j} S_{(i, j)}^{I}
$$

We normalize $S_{i}^{I}$ with softmax afterwards. Then we obtain representations for each role $r_{k}$ in Eq. 14 .

$$
h_{(A \rightarrow B)}^{r_{k}}=\sum_{i=1}^{N}\left(S_{i}^{I} h_{(A \rightarrow B)_{i}}^{r_{k}}\right) .
$$




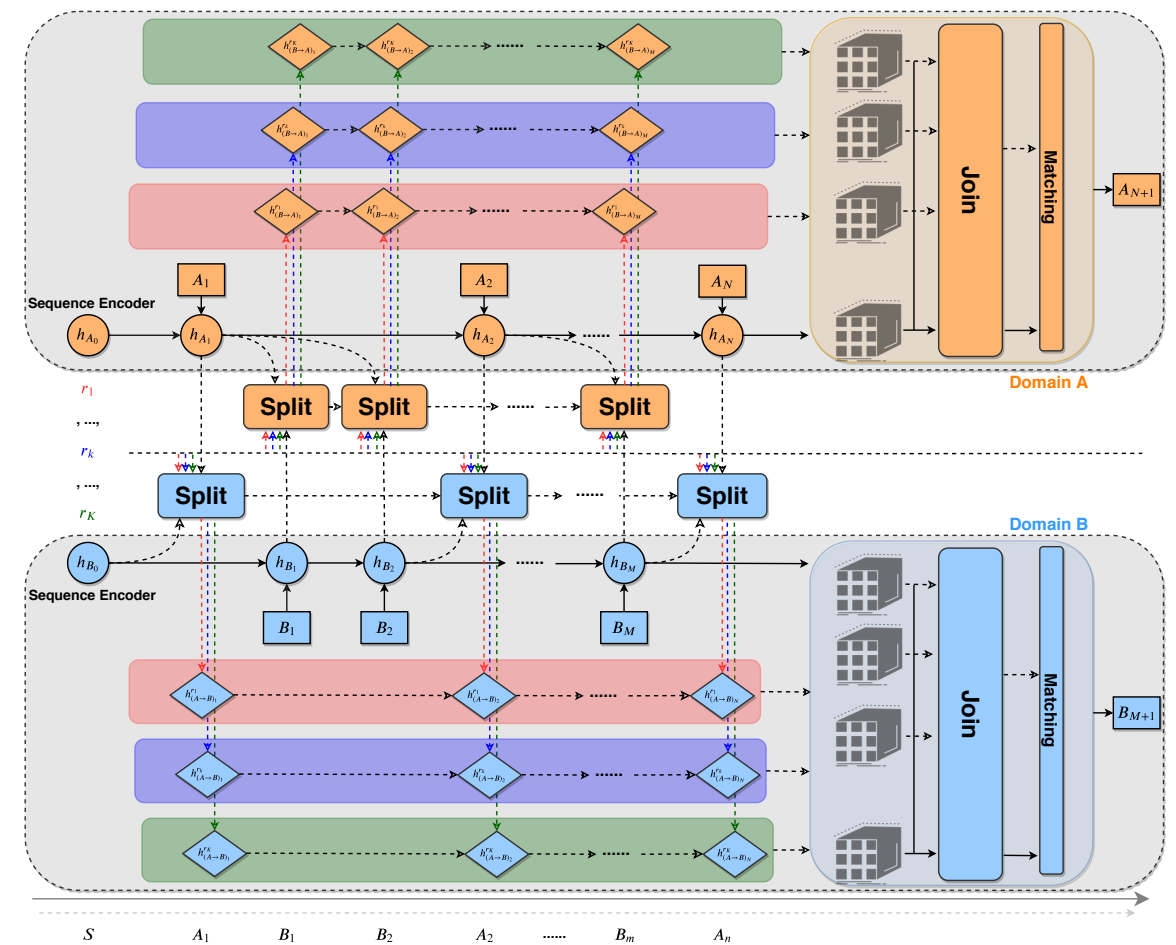

Fig. 4: An overview of PSJNet-II. As before, the orange and blue colors represent different domains. Red, purple and green represent different user roles. Section III-D contains a walkthrough of the model.

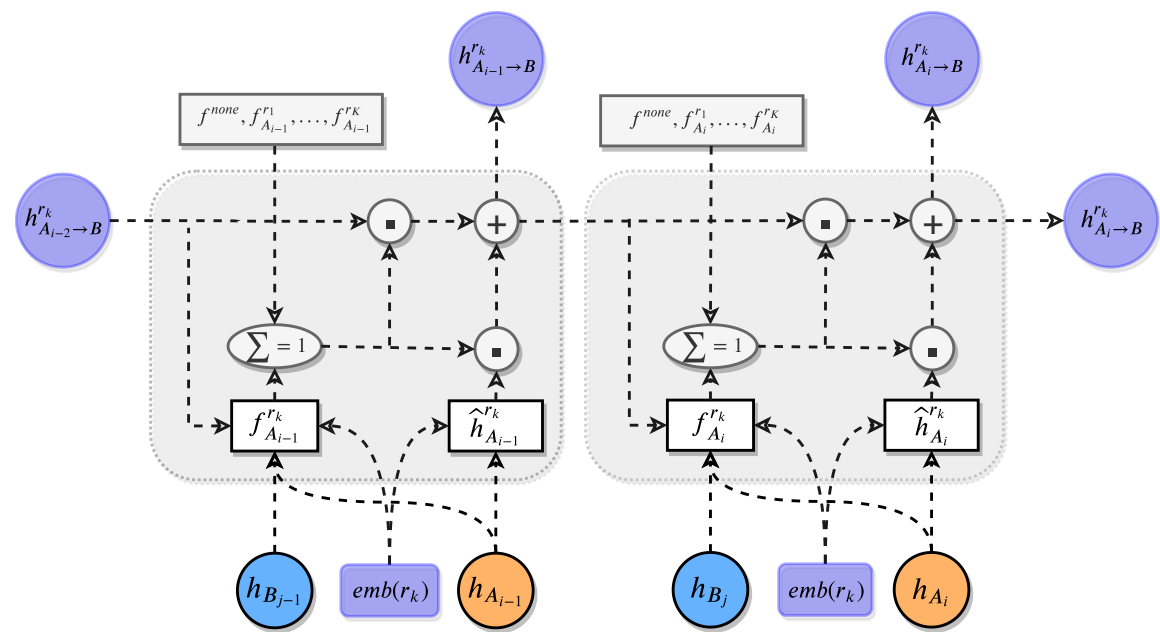

Fig. 5: The split unit for $r_{k}$ illustrated while transforming information from domain $A$ to domain $B$.

Finally, we get the cross-domain representation $h_{(A \rightarrow B)}$ by joining $\left\langle h_{(A \rightarrow B)}^{r_{1}}, \ldots, h_{(A \rightarrow B)}^{r_{K}}\right\rangle$ again with similar operations as in 12 and 14 , but with a different similarity matrix $S^{I I} \in \mathbb{R}^{M \times K}$. Note that $S^{I I}$ is computed between $\left\langle h_{(A \rightarrow B)}^{r_{1}}, \ldots, h_{(A \rightarrow B)}^{r_{K}}\right\rangle$ and $\left\langle h_{B_{1}}, \ldots, h_{B_{M}}\right\rangle$ this time.

There are two strengths of PSJNet-II compared to PSJNet-I. First, the normalization (see Eq. 10 reduces the influence of some large gate values, thereby making the prediction more accurate. Second, the split-by-join unit of PSJNet-I uses the output of the last time step of GRU as the cross-domain representation from domain $A$ to domain $B$. Information in the intermediate step is lost to some degree. However, in the join unit of PSJNet-II, the cross-domain representation from A to B undergoes more fine-grained calculations.

\section{E. Hybrid recommendation decoder}

The hybrid recommendation decoder integrates hybrid information from both domains $A$ and $B$ to evaluate the recommendation probabilities of the candidate items. Specifically, it first gets the hybrid representation by concatenating the representation $h_{B}$ from domain $B$ and the transformed representation $h_{(A \rightarrow B)}$ from domain $A$ to domain $B$. Then, it evaluates the recommendation probability for each candidate item by calculating the matching of between the hybrid representation and the item-embedding matrix followed by a softmax function, as shown in Eq. 15 .

$$
P\left(B_{j+1} \mid S\right)=\operatorname{softmax}\left(W_{I} \cdot\left[h_{B}, h_{(A \rightarrow B)}\right]^{\top}+b_{I}\right),
$$




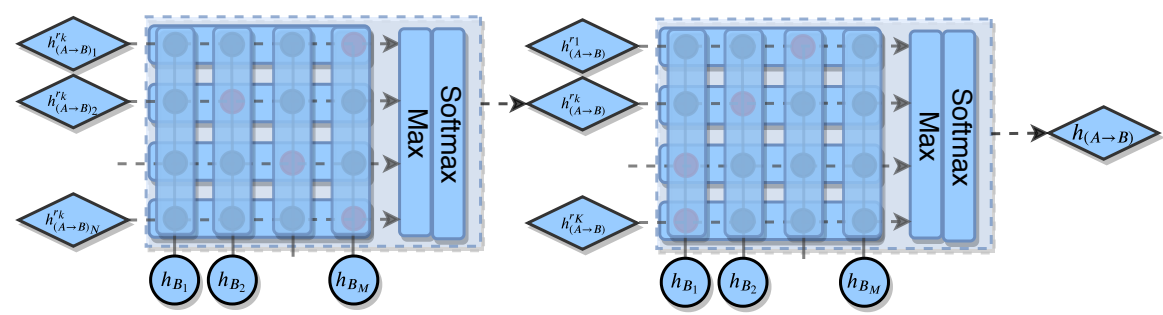

Fig. 6: The join unit illustrated while transforming information from domain $A$ to domain $B$.

where $W_{I}$ is the embedding matrix of all items of domain $B$; $b_{I}$ is the bias term.

\section{F. Objective function}

We employ the negative log-likelihood loss function to train PSJNet in each domain as follows:

$$
\begin{aligned}
& L_{A}(\theta)=-\frac{1}{|\mathbb{S}|} \sum_{S \in \mathbb{S}} \sum_{A_{i} \in S} \log P\left(A_{i+1} \mid S\right) \\
& L_{B}(\theta)=-\frac{1}{|\mathbb{S}|} \sum_{S \in \mathbb{S}} \sum_{B_{j} \in S} \log P\left(B_{j+1} \mid S\right),
\end{aligned}
$$

where $\theta$ are all the parameters of our model PSJNet and $\mathbb{S}$ are the sequence instances in the training set. In the case of joint learning, the final loss is a linear combination of both losses:

$$
L(\theta)=L_{A}(\theta)+L_{B}(\theta) .
$$

All parameters as well as the item embeddings are learned in an end-to-end back-propagation training way.

\section{EXPERIMENTAL SETUP}

\section{A. Research questions}

We seek to answer the following research questions in our experiments:

(RQ1) What is the performance of PSJNet-I and PSJNet-II on the SAC-SR task? Do they outperform the state-ofthe-art methods in terms of Recall and MRR?

(RQ2) Which PSJNet variant is more effective in the SAC-SR task? PSJNet-I or PSJNet-II? What are the performances of different groups of methods, e.g., sequential and non-sequential recommendation methods?

(RQ3) What is the performance of PSJNet-I and PSJNetII on different domains and different datasets? Do they improve the performance of both domains and datasets? Are the improvements equivalent?

\section{B. Datasets}

We need datasets that exhibit both share-account and crossdomain characteristics to conduct experiments. To demonstrate the effectiveness of the proposed PSJNet model, we build and release two new datasets, HAmazon and HVIDEO, respectively. We build the HAmazon dataset by simulating shared account characteristics using previously released Amazon datasets. HVIDEO has previously been used in [52] but we release it with this paper. Details of the two datasets are as follows.
- HAmazon: He and McAuley [26] have released an Amazon product dataset that contains product reviews (ratings, text, helpfulness votes) and metadata (descriptions, category information, price, brand, and image features) from Amazon; it includes 142.8 million reviews spanning the period May 1996-July 2014. The data contains user behavior from multiple domains. In this paper, we use data from two Amazon domains. The M-domain contains movie watching and rating behavior of Amazon users. The B-domain covers book reading and rating behavior of Amazon users. We collect user-id, item-id, rating, and timestamp from the data and conduct some preprocessing. We first order the items by time under each account. Then, we merge records of the same item watched/read by the same user with an adjacent timestamp. We only keep items whose frequency is larger than 5 in the M-domain and 10 in the B-domain.

To satisfy cross-domain characteristics, we first find users whose behavior can be found in both the Amazon movie and book domains and then only keep users who have more than 10 records.

To simulate shared account characteristics, we first split the data into 6 consecutive intervals, 1996-2000, 2001-2003, 2004-2006, 2007-2009, 2010-2012, and 2013-2015. Then, we combine data from both domains by randomly merging 2,3 , or 4 users and their behavior in each interval as one shared account. Because each sequence has a lot of user behavior recorded over a long period of time, we split the sequences from each account into several small sequences with each containing watching/reading records within a year. We also require that each sequence contains at least 5 items from the M-domain and 2 items from the B-domain. The length of each sequence is between 4 and 60 with an average length of 31.29 .

For evaluation, we use the last watched/read item in each sequence for each domain as the ground truth respectively. We randomly select $75 \%$ of all data as the training set, $15 \%$ as the validation set, and the remaining $10 \%$ as the test set. The statistics of the final dataset are shown in Table [] Note that although HAmazon can be used for experiments, it is not a SAC-SR dataset by nature. There are two shortcomings. First, the merged users do not naturally have the shared account characteristic. Second, the two domains are quite different and are not well correlated in content, which means that the items in different domains have little chance to reflect similar interests.

- HVIDEO: To facilitate future research for SAC-SR, we also release another dataset, HVIDEO, which exhibits 
TABLE I: Statistics of the datasets.

\begin{tabular}{|c|c|c|c|}
\hline \multicolumn{2}{|c|}{ HAmazon } & \multicolumn{2}{|c|}{ HVIDEO } \\
\hline M-domain & & $V$-domain & \\
\hline \#Items & 67,161 & \#Items & 16,407 \\
\hline$\# \operatorname{Logs}$ & $4,406,924$ & $\# \operatorname{Logs}$ & 227,390 \\
\hline$B$-domain & & E-domain & \\
\hline \#Items & 126,547 & \#Items & 3,380 \\
\hline \#Logs & $4,287,240$ & \#Logs & 177,758 \\
\hline \#Overlapped-users & 13,724 & \#Overlapped-users & 13,714 \\
\hline \#Sequences & 289,160 & \#Sequences & 134,349 \\
\hline \#Training-sequences & 204,477 & \#Training-sequences & 102,182 \\
\hline \#Validation-sequences & 49,814 & \#Validation-sequences & 18,966 \\
\hline \#Test-sequences & 34,869 & \#Test-sequences & 13,201 \\
\hline
\end{tabular}

shared-account and cross-domain characteristics by nature. HVIDEO is a smart TV dataset that contains watching logs of $260 \mathrm{k}$ users from October 1st 2016 to June 30th 2017. The logs are collected on two platforms (the V-domain and the E-domain) from a well-known smart TV service provider. The V-domain contains family video watching behavior including TV series, movies, cartoons, talent shows and other programs. The E-domain covers online educational videos based on textbooks from elementary to high school, as well as instructional videos on sports, food, medical, etc. On the two platforms, we gather user behavior, including which video is played, when a smart TV starts to play a video, and when it stops playing the video, and how long the video has been watched. Compared with previous datasets, HVIDEO contains rich and natural family behavior data generated in a natural shared account and cross-domain context. Therefore, it is very suitable for SAC-SR research. We conduct some preprocessing on the dataset. We first filter out users who have less than 10 watching records and whose watching time is less than 300 seconds. Then, we merge records of the same item watched by the same user with an adjacent time less than 10 minutes. After that, we combine data from different domains together in chronological order which is grouped by the same account. Because each account has a lot of logs recorded in a long time, we split the logs from each account into several small sequences with each containing 30 watching records. And we require that the number of items in both domains must be greater than 5 in each sequence, which can ensure the sequences mix is high enough.

For evaluation, we use the last watched item in each sequence for each domain as the ground truth, respectively. We randomly select $75 \%$ of all data as the training set, $15 \%$ as the validation set, and the remaining $10 \%$ as the test set. The statistics of the final dataset are shown in Table []

\section{Baseline methods}

For our contrastive experiments, we consider baselines from four categories: traditional, sequential, shared account, and cross-domain recommendations.

1) Traditional recommendations.

As traditional recommendation methods, we consider the following:
- POP: This method ranks items in the training set based on their popularity, and always recommends the most popular items. It is a very simple baseline, but it can perform well in certain domains and is frequently used as a baseline because of its simplicity [28].

- Item-KNN: The method computes a degree of item-to-item similarity that is defined as the number of co-occurrences of two items within sequences divided by the square root of the product of the number of sequences in which either item occurs. Items that are similar to the actual item will be recommended by this baseline. Regularization is included to avoid coincidental high similarities between rarely visited items [46].

- BPR-MF: This model is a commonly used matrix factorization method. This model cannot be applied directly to SRs, because new sequences do not have pre-computed feature vectors. Like [30], we apply it for sequential recommendations by representing a new sequence with the average latent factors of items that appeared in this sequence, i.e., we average the similarities of the feature vectors between a recommendable item and the items of the session so far.

\section{2) Shared account recommendations.}

There are some studies that explore shared account recommendations by first achieving user identification [38, 81, 3]. However, they need extra information for user identification, e.g., some explicit ratings for movies or descriptions for some songs, even some textual descriptions for flight tickets, which is not available in our datasets. Therefore, we select a method that works on the IP-TV recommendation task that is similar to ours.

- VUI-KNN: This model encompasses an algorithm to decompose members in a composite account by mining different user interests over different time periods from logs [77]. The method first divides a day into time periods, so the logs of each account fall into the corresponding time period; $\operatorname{logs}$ in each time period are assumed to be generated by a virtual user that is represented by a 3-dimensional $\{$ account $\times$ item $\times$ time $\}$ vector. After that, cosine similarity is used to calculate similarity among virtual users, some of which are merged into a latent user. VUI deploys the UserKNN method to produce recommendations for these latent users. 


\section{3) Cross-domain recommendations.}

For cross-domain recommendations, we choose two baseline methods.

- NCF-MLP++: This model uses a deep learning-based process to learn the inner product of the traditional collaborative filtering by using a multilayer perceptron (MLP) [28]. We improve NCF-MLP by sharing the collaborative filtering in different domains. It is too time-consuming to rank all items with this method, because it needs to compute a score for each item one by one. We randomly sample four negative instances for each positive instance in the training process, and sample 3,000 negatives for each predicted target item in the test process, thus simplifying the task for this method.

- Conet: This is a neural transfer model across domains on the basis of a cross-stitch network [32, 55], where a neural collaborative filtering model [28] is employed to share information between domains.

\section{4) Sequential recommendations.}

Recently, a number of sequential recommendations methods have been proposed; RNN-based neural methods have outperformed traditional MC- or MDP-based methods. There are many RNN-based methods. In this paper, we consider two methods with somewhat similar architectures as PSJNet.

- GRU4REC: This model uses a GRU to encode sequential information. It uses a session-parallel mini-batch training process and employs ranking-based loss functions for learning the model [30].

- HGRU4REC: Quadrana et al. [59] propose this model based on RNNs which can deal with two cases: (1) user identifiers are present and propagate information from the previous sequence (user session) to the next, thus improving the recommendation accuracy, and (2) there are no past sessions (i.e., no user identifiers). The model is based on a hierarchical RNN, where the hidden state of a lowerlevel RNN at the end of one sequence is passed as input to a higher-level RNN, which is meant to predict a good initialization for the hidden state of the lower RNN for the next sequence.

\section{Evaluation metrics}

Recommender systems can only recommend a limited number of items at a time. The item a user might pick should be amongst the first few in the ranked list [59, 13, 29]. Commonly used metrics are MRR@20 and Recall@20 [44, 61, 54]. In this paper,we also report MRR@5, Recall@5 and MRR@10, Recall@10.

- Recall: The primary evaluation metric is Recall, which measures the proportion of cases when the relevant item is amongst the top ranked items in all test cases. Recall does not consider the actual rank of the item as long as it is amongst the recommendation list. This accords with certain real-world scenarios well where there is no highlighting of recommendations and the absolute order does not matter [30].

- MRR: Another used metric is MRR (Mean Reciprocal Rank), which is the average of reciprocal ranks of the relevant items. And the reciprocal rank is set to zero if the ground truth item is not in the list of recommendations. MRR takes the rank of the items into consideration, which is vital in settings where the order of recommendations matters. We choose MRR instead of other ranking metrics, because there is only one ground truth item for each recommendation; no ratings or grade levels are available.

For significance testing we use a paired t-test with $p<0.05$.

\section{E. Implementation details}

We set the item embedding size and GRU hidden state size to 90 . We use dropout [67] with drop ratio $p=0.8$. These settings are chosen with grid search on the validation set. For the latent user size $K$, we try different settings, an analysis of which can be found in Section VI-B. We initialize the model parameters randomly using the Xavier method [25]. We take Adam as our optimizing algorithm. For the hyper-parameters of the Adam optimizer, we set the learning rate $\alpha=0.001$. We also apply gradient clipping [58] with range $[-5,5]$ during training. To speed up the training and converge quickly, we use mini-batch size 64. We test the model performance on the validation set for every epoch. Both PSJNet-I and PSJNet-II are implemented in Tensorflow and trained on a GeForce GTX TitanX GPU.

\section{EXPERIMENTAL RESULTS}

To answer RQ1, RQ2 and RQ3, we report the results of PSJNet compared with the baseline methods on the HAmazon and HVIDEO datasets, as shown in Table III and III respectively. From the tables, we can see that both PSJNetI and PSJNet-II outperform all baselines in terms of MRR and Recall for all reported values. Below, we discuss several insights we obtain from Table [II and III] so as to answer our research questions.

\section{A. Overall performance on the SAC-SR task (RQ1)}

Both two PSJNet variants significantly outperform all baselines and achieve the best results on all metrics, including strong baselines, i.e., GRU4REC and HGRU4REC. It is worth noting that although recent studies on SR propose many neural network models, we choose GRU4REC and HGRU4REC because GRU4REC and HGRU4REC have very similar architectures as PSJNet. And to obtain a fair comparison, we re-implement them within the same TensorFlow framework as we use for PSJNet.

Specifically, on the HVIDEO dataset, the largest increase of PSJNet-II over GRU4REC is 4.04\% in terms of MRR@20, and $4.48 \%$ in terms of Recall@10 on the V-domain. On the E-domain, the increase is even larger with a $4.70 \%$ increase of PSJNet-II over GRU4REC in terms of MRR@20 and 13.03\% increase of PSJNet-I over GRU4REC in terms of Recall@20. And the increase over HGRU4REC on the V-domain is $1.69 \%$ and $3.45 \%$ (at most) in terms of MRR and Recall respectively. On the E-domain, the increase is $2.29 \%$ and $7.67 \%$ respectively. We believe that those performance improvements are due to the fact that PSJNet considers two important factors (shared-account and cross-domain) with its parallel modeling 
TABLE II: Experimental results (\%) on the HAmazon dataset.

\begin{tabular}{|c|c|c|c|c|c|c|c|c|c|c|c|c|}
\hline \multirow{3}{*}{ Methods } & \multicolumn{6}{|c|}{ M-domain recommendation } & \multicolumn{6}{|c|}{ B-domain recommendation } \\
\hline & \multicolumn{3}{|c|}{ MRR } & \multicolumn{3}{|c|}{ Recall } & \multicolumn{3}{|c|}{ MRR } & \multicolumn{3}{|c|}{ Recall } \\
\hline & @5 & @ 10 & @20 & @5 & $@ 10$ & @ 20 & @ 5 & @ 10 & $@ 20$ & @5 & $@ 10$ & @ 20 \\
\hline POP & 0.36 & 0.44 & 0.49 & 0.73 & 1.32 & 2.02 & 0.14 & 0.19 & 0.22 & 0.42 & 0.78 & 1.22 \\
\hline Item-KNN & 1.28 & 1.57 & 1.86 & 2.58 & 4.83 & 9.00 & 3.23 & 3.94 & 4.55 & 6.65 & 12.11 & 20.94 \\
\hline BPR-MF & 2.90 & 3.00 & 3.06 & 3.90 & 4.65 & 5.50 & 0.88 & 0.92 & 0.96 & 1.23 & 1.50 & 2.15 \\
\hline VUI-KNN & - & - & - & - & - & - & - & - & - & - & - & - \\
\hline NCF-MLP++ & 13.68 & 13.96 & 14.21 & 18.44 & 20.58 & 24.31 & 13.67 & 13.90 & 14.05 & 18.14 & 19.92 & 22.08 \\
\hline Conet & 14.64 & 14.90 & 15.12 & 19.25 & 21.25 & 24.46 & 15.85 & 16.09 & 16.28 & 20.98 & 22.83 & 25.56 \\
\hline GRU4REC & 82.01 & 82.08 & 82.11 & 83.10 & 83.61 & 84.06 & 81.34 & 81.41 & 81.44 & 82.77 & 83.32 & 83.76 \\
\hline HGRU4REC & 83.07 & 83.12 & 83.14 & 84.24 & 84.65 & 84.91 & 82.15 & 82.26 & 82.31 & 83.46 & 84.30 & 84.91 \\
\hline PSJNet-I & 83.91 & 83.94 & 83.95 & 84.91 & 85.13 & 85.33 & 84.93 & 84.93 & 84.93 & 85.33 & 85.36 & 85.38 \\
\hline PSJNet-II & $84.01^{\dagger}$ & $84.04^{\dagger}$ & $84.05^{\dagger}$ & 84.88 & 85.10 & 85.28 & $\mathbf{8 5 . 1 0}{ }^{\dagger}$ & $\mathbf{8 5 . 1 0}^{\dagger}$ & $85.11^{\dagger}$ & 85.32 & 85.37 & 85.38 \\
\hline
\end{tabular}

Bold face indicates the best result in terms of the corresponding metric. Significant improvements over the best baseline results are marked with ${ }^{\dagger}$ (t-test, $p<.05$ ). To ensure a fair comparison, we re-implemented GRUE4REC and HGRU4REC in Tensorflow, just like PSJNet; the final results may be slightly different from the Theano version released by the authors. To obtain the results of NCF-MLP++ and Conet, we run the code released by $\mathrm{Hu}$ et al. [32]. VUI-KNN does not work on this dataset because it needs specific time in a day which is not available on the HAmazon dataset.

TABLE III: Experimental results (\%) on the HVIDEO dataset.

\begin{tabular}{|c|c|c|c|c|c|c|c|c|c|c|c|c|}
\hline \multirow{3}{*}{ Methods } & \multicolumn{6}{|c|}{ V-domain recommendation } & \multicolumn{6}{|c|}{ E-domain recommendation } \\
\hline & \multicolumn{3}{|c|}{ MRR } & \multicolumn{3}{|c|}{ Recall } & \multicolumn{3}{|c|}{ MRR } & \multicolumn{3}{|c|}{ Recall } \\
\hline & @5 & $@ 10$ & $@ 20$ & @5 & $@ 10$ & $@ 20$ & @5 & @ 10 & $@ 20$ & @5 & @ 10 & @20 \\
\hline POP & 2.66 & 3.07 & 3.27 & 5.01 & 7.77 & 10.49 & 1.71 & 1.96 & 2.24 & 2.21 & 3.61 & 6.58 \\
\hline Item-KNN & 4.43 & 4.16 & 2.93 & 10.48 & 16.49 & 23.93 & 2.11 & 2.39 & 2.90 & 3.01 & 5.77 & 12.11 \\
\hline BPR-MF & 1.21 & 1.31 & 1.36 & 1.88 & 2.56 & 3.38 & 1.34 & 1.52 & 1.64 & 2.74 & 4.05 & 5.83 \\
\hline VUI-KNN & 3.44 & 3.53 & 2.87 & 16.46 & 24.85 & 34.76 & 2.03 & 2.51 & 3.48 & 6.36 & 11.55 & 24.27 \\
\hline NCF-MLP++ & 16.25 & 17.25 & 17.90 & 26.10 & 33.61 & 43.04 & 3.92 & 4.57 & 5.14 & 7.36 & 12.27 & 20.84 \\
\hline Conet & 21.25 & 22.61 & 23.28 & 32.94 & 43.07 & 52.72 & 5.01 & 5.63 & 6.21 & 9.26 & 14.07 & 22.71 \\
\hline GRU4REC & 78.27 & 78.46 & 78.27 & 80.15 & 81.63 & 83.04 & 12.27 & 13.00 & 13.70 & 16.24 & 21.89 & 32.16 \\
\hline HGRU4REC & 80.37 & 80.53 & 80.62 & 81.92 & 83.21 & 84.43 & 14.47 & 15.37 & 16.11 & 19.79 & 26.72 & 37.52 \\
\hline PSJNet-I & 80.51 & 80.80 & 80.95 & 83.22 & 85.34 & 87.48 & 14.63 & 15.83 & 16.88 & 20.41 & 29.61 & 45.19 \\
\hline PSJNet-II & $81.97^{\dagger}$ & $82.20^{\dagger}$ & $82.32^{\dagger}$ & $84.32^{\dagger}$ & $86.11^{\dagger}$ & $\mathbf{8 7 . 7 5}{ }^{\dagger}$ & $16.63^{\dagger}$ & $17.62^{\dagger}$ & $18.46^{\dagger}$ & $22.12^{\dagger}$ & 29.64 & 42.20 \\
\hline
\end{tabular}

The same conventions are used as in Table

architecture and two main components for as part of its end-to-end recommendation model, namely the "split" and "join". With these three modules, PSJNet is able to model user interests more accurately by leveraging complementary information from both domains and improve recommendation performance in both domains. We will analyze the effects of the three modules in more depth in Section VI-A

\section{B. Comparing two versions of PSJNet with different groups} of methods (RQ2)

Generally, PSJNet-II outperforms PSJNet-I on both datasets. Specifically, PSJNet-II outperforms PSJNet-I in terms of most metrics on both domains on the HVIDEO dataset, especially for MRR@5 and Recall@5. The performance is comparable on the HAmazon dataset. But as we mentioned in \$IV-B. HAmazon is not a good dataset for SAC-SR because the shared-account characteristic is simulated, and the two domains are quite different and not well correlated in content. Since both PSJNet-I and PSJNet-II adopt the parallel modeling architecture, we can conclude that the superiority of PSJNetII over PSJNet-I mainly comes from the separate modeling of "split" and "join". We will show this in more depth in Section VI-A

We can also see that RNN-based methods (e.g., GRU4REC, HGRU4REC, and our PSJNet) perform much better than traditional methods, which demonstrates that RNN-based methods are good at dealing with sequential information. They are able to learn better dense representations of the data through nonlinear modeling, which we assume is able to provide a higher level of abstraction. The shared account and cross-domain baselines (e.g., VUI-KNN, NCF-MLP++ and Conet) perform much worse than PSJNet. They also perform substantially worse than HGRU4REC. This is because these shared account and cross-domain baselines ignore sequential information; VUI-KNN only considers the length of watching time, and NCF-MLP++ and Conet do not use any time information. Another reason is that NCF-MLP++ and Conet just map each overlapped account in both domains to the same latent space to calculate the user-item similarity. However, the existence of shared accounts makes it difficult to find the same latent space for different latent user roles under one account. Besides, VUI$\mathrm{KNN}$ is not a deep learning method and it simply distinguishes 
user roles based on the fixed divided time periods in a day, which means it assumes there is only one family member in each time period. However, in the smart TV scenario, many people usually watch programs together [77]. This situation cannot be captured very well by VUI-KNN. And it requires the specific time of the user behaviors in a day in order to distinguish different user roles. That is why we cannot use it to obtain results on the HAmazon dataset because there is no such information. In contrast, PSJNet can extract components of each hidden user role according to their viewing records in another domain with the "split" module. The results of BPR-MF are lower than of POP, which indicates that most items users watched are very popular, which is in line with the phenomenon that people like to pursue popular items in the video and book recommendation scenarios.

\section{Contrasting the performance on different domains and different datasets (RQ3)}

The Recall values of PSJNet on the HAmazon dataset are comparable on the two domains while the Recall values on the V-domain are higher than those on the E-domain on the HVIDEO dataset. This is also true for the other methods. We believe that this is because of data balance issues. On the HAmazon dataset, the data is generally balanced on two domains. Most accounts have an equal amount of data on both domains. This means that the models can learn pretty well with data from just one domain. Cross-domain information is not that important: the increase of PSJNet on the HAmazon dataset is relatively small. However, the situation is different on the HVIDEO dataset. Most accounts have much more data on the V-domain due to its content diversity; because of this, models can better learn user's viewing characteristics on the V-domain. Therefore, on the HAmazon dataset, the space for improvement on both domains is smaller than on the HVIDEO dataset.

Additionally, comparing PSJNet with the best baseline, HGRU4REC, we find that the largest increase on the E-domain is larger than on the V-domain. The largest increase in MRR is $1.69 \%$ on the V-domain and $2.29 \%$ on the E-domain. And for the Recall values, the largest increase is $3.45 \%$ on the V-domain, and $7.67 \%$ on the E-domain. This shows that the space for potential improvements on the V-domain is smaller than on the E-domain after considering shared account and cross-domain information.

Also, the increases in MRR and Recall are different on two datasets. On the HAmazon dataset, there is no significant difference for both MRR and Recall from @5 to @20. This means that PSJNet can already predict the ground truth item within the top-5 for most cases. This is not true on the HVIDEO dataset, especially on the E-domain. Specifically, the largest increase is $2.25 \%$ for MRR from the top-5 to the top20 , and $24.78 \%$ for Recall.

\section{ANALYSIS}

\section{A. Ablation study}

In this subsection, we report on an ablation study to verify how well the parallel modeling schema, with the "split" and "join" units, improves the recommendation performance. The results are shown in Table $\overline{I V}$ and $\mathrm{V}$. PSJNet (-PSJ) is the PSJNet-I or PSJNet-II without all the three parts, which degenerates into GRU4REC except that PSJNet (-PSJ) is jointly trained on two domains. PSJNet-I (-SJ) is PSJNet-I without "split-by-join" unit. PSJNet-II (-S) is PSJNet-II without the "split" unit and PSJNet-II (-J) is PSJNet-II without the "join" unit (i.e., replacing the "join" unit by summing up the outputs from the "split" unit). We can draw the following conclusions from the results.

First, almost all the best results are almost all from PSJNetI and PSJNet-II, which demonstrates the effectiveness of combining all three parts. The three parts bring around 7\% (MRR) and 1\%-3\% (Recall) improvements on the M-domain of HAmazon, and around 4\% (MRR) and 4\%-10\% (Recall) on both domains of HVIDEO. Additionally, we see that PSJNet (-PSJ) gets the lowest performance amongst these methods, while it still outperforms most of the baselines listed in Table III and III In summary, then, combining information from an auxiliary domain is useful. The MRR improvements are larger on HAmazon while the Recall improvements are larger on HVIDEO. This is due to the different characteristics of different domains. Take the two domains in HVIDEO for example. Almost all members have viewing records in the V-domain. However, items on the E-domain are mostly educational programs, so children take up a large proportion, and their educational interests are relatively fixed. As a result, the information extracted from the V-domain mostly belongs to children, which is less helpful because we already have enough data on the E-domain to learn such features in most cases.

Second, generally parallel modeling brings the most improvements followed by the "split" and "join" units. Specifically, PSJNet-I achieves around 5\% (MRR) and 2\% (Recall) improvements on the M-domain of HAmazon with the parallel modeling while further improvements with the "split-by-join" unit are just around $0.6 \%$ (MRR) and 1\% (Recall). Similar results can be found on the B-domain of HAmazon and Edomain of HVIDEO. We believe this is because the model is already able to leverage information from both domains to achieve recommendations with the parallel modeling schema. It is further improved by taking other factors, e.g., sharedaccount characteristics, into account in order to better leverage the cross-domain information. This is why the "split" and "Joint" units are able to further improve the results over the parallel modeling schema. An exception is that the "split" and "join" units achieve more improvements than the parallel modeling on the V-domain of HVIDEO for PSJNet-I. We think the reason is that PSJNet-I (-SJ) cannot effectively use the cross-domain information without the "split-by-join" unit, while PSJNet-II (-S) is better because the function of "split" unit is replaced by the "join" unit to some extent. The same is true for PSJNet-II (-J). This could be verified by the fact that both PSJNet-I and PSJNet-II get big improvements with both units than with neither, but the improvements are smaller than with one unit for PSJNet-II.

Third, the "split" unit is generally more effective than the "join" unit for PSJNet-II as we find that the gap between 
TABLE IV: Analysis of the contribution of the parallel modeling, split unit and join unit on the HAmazon dataset.

\begin{tabular}{|c|c|c|c|c|c|c|c|c|c|c|c|c|}
\hline \multirow{3}{*}{ Methods } & \multicolumn{6}{|c|}{ M-domain recommendation } & \multicolumn{6}{|c|}{ B-domain recommendation } \\
\hline & \multicolumn{3}{|c|}{ MRR } & \multicolumn{3}{|c|}{ Recall } & \multicolumn{3}{|c|}{ MRR } & \multicolumn{3}{|c|}{ Recall } \\
\hline & @5 & @ 10 & @20 & @5 & @ 10 & $@ 20$ & @5 & $@ 10$ & $@ 20$ & @5 & @ 10 & @20 \\
\hline PSJNet (-PSJ) & 77.26 & 77.44 & 77.51 & 82.22 & 83.52 & 84.39 & 81.69 & 81.72 & 81.73 & 85.03 & 85.27 & 85.34 \\
\hline PSJNet-I (-SJ) & 83.30 & 83.32 & 83.33 & 84.03 & 84.20 & 84.31 & 84.04 & 84.04 & 84.04 & 85.31 & 85.35 & 85.38 \\
\hline PSJNet-II (-S) & 83.55 & 83.59 & 83.60 & 84.61 & 84.90 & 85.14 & 84.87 & 84.88 & 84.88 & 85.26 & 85.31 & 85.35 \\
\hline PSJNet-II (-J) & 82.28 & 82.35 & 82.38 & 84.02 & 84.52 & 84.92 & 83.42 & 83.45 & 83.46 & 84.79 & 84.96 & 85.08 \\
\hline PSJNet-I & 83.91 & 83.94 & 83.95 & 84.91 & 85.13 & 85.33 & 84.93 & 84.93 & 84.93 & 85.33 & 85.36 & 85.38 \\
\hline PSJNet-II & 84.01 & 84.04 & 84.05 & 84.88 & 85.10 & 85.28 & 85.10 & 85.10 & 85.11 & 85.32 & 85.37 & 85.38 \\
\hline
\end{tabular}

PSJNet (-PSJ) is PSJNet without parallel modeling, i.e., no cross-domain representations are used for recommendations. Without parallel modeling, both PSJNet-I and PSJNet-II become the same PSJNet (-PSJ). PSJNet-I $(-\mathrm{SJ})$ is PSJNet-I without "split-by-join" unit. Because "split-by-join" is an indivisible unit, there is no PSJNet-I $(-S)$ or PSJNet-I (-J). PSJNet-II (-S) is PSJNet-II without the "split" unit and PSJNet-II (-J) is PSJNet-II without the "join" unit.

TABLE V: Analysis of the contribution of the parallel modeling, split unit and join unit on the HVIDEO dataset.

\begin{tabular}{|c|c|c|c|c|c|c|c|c|c|c|c|c|}
\hline \multirow{3}{*}{ Methods } & \multicolumn{6}{|c|}{ V-domain recommendation } & \multicolumn{6}{|c|}{ E-domain recommendation } \\
\hline & \multicolumn{3}{|c|}{ MRR } & \multicolumn{3}{|c|}{ Recall } & \multicolumn{3}{|c|}{ MRR } & \multicolumn{3}{|c|}{ Recall } \\
\hline & @5 & @ 10 & @ 20 & @5 & @ 10 & @ 20 & @5 & @ 10 & @ 20 & @ 5 & @ 10 & @20 \\
\hline PSJNet (-PSJ) & 78.02 & 78.17 & 78.28 & 80.13 & 81.34 & 82.93 & 12.69 & 13.43 & 14.05 & 16.54 & 22.29 & 31.45 \\
\hline PSJNet-I (-SJ) & 78.59 & 78.85 & 78.97 & 81.71 & 83.58 & 85.33 & 16.35 & 17.04 & 17.59 & 20.97 & 26.29 & 34.44 \\
\hline PSJNet-II (-S) & 81.61 & 81.85 & 81.99 & 83.93 & 85.73 & 87.71 & 15.94 & 17.01 & 17.84 & 20.96 & 29.18 & 41.38 \\
\hline PSJNet-II (-J) & 81.76 & 81.98 & 82.12 & 84.20 & 85.80 & 87.77 & 16.43 & 17.48 & 18.46 & 21.92 & 29.96 & 44.30 \\
\hline PSJNet-I & 80.51 & 80.80 & 80.95 & 83.22 & 85.34 & 87.48 & 14.63 & 15.83 & 16.88 & 20.41 & 29.61 & 45.19 \\
\hline PSJNet-II & 81.97 & 82.20 & 82.32 & 84.32 & 86.11 & 87.75 & 16.63 & 17.62 & 18.46 & 22.12 & 29.64 & 42.20 \\
\hline
\end{tabular}

The same settings are applied as in Table IV

PSJNet-II and PSJNet-II (-J) is smaller than between PSJNet-II and PSJNet-II (-S). On the one hand, this shows that the "split" unit plays a more important role which addresses the challenge raised by shared accounts, i.e., filtering out information of some user roles that might be useful for another domain from the mixed user behaviors. On the other hand, the results also show that the current "join" unit is not effective enough as directly summing up the outputs from the "split" unit also achieves competitive performance, and/or the improvement space of the "join" unit is limited.

\section{B. Influence of the hyperparameter $K$}

Both PSJNet-I and PSJNet-II introduce a hyperparameter $K$ in the "split" unit which corresponds to the number of latent user roles. We carry out experiments to study how setting $K$ affects the recommendation performance of PSJNet on both datasets, and whether the best $K$ is the same under all situations and accords with reality. Taking into account common sizes of families, we consider $K=1, \ldots, 5$, and compare different values of $K$ while keeping other settings unchanged. The results are shown in Table VI and VII

First, we see that the best values in terms of MRR and Recall are achieved when $K=3,4, K=4$ for PSJNet-I and $K=3$ for PSJNet-II especially. This is consistent with the size of modern families on HVIDEO and the simulation settings on HAmazon. For PSJNet-I, the lowest MRR and Recall values are achieved when $K=1$. But for PSJNetII, the gap between the best and worst performances is much smaller, which indicates that PSJNet-II is less sensitive to $K$ than PSJNet-I.
Seond, both PSJNet-I and PSJNet-II show mostly consistent trends and conclusions on both datasets, i.e., the best $K$ values are basically the same. On the one hand, this demonstrates the performance stability of both PSJNet-I and PSJNet-II. On the other hand, this is also a clue that both models identify $K$ as the potential user roles under each account, which verifies our assumption.

Third, although $K$ can affect the recommendation performance, the influence is limited. As we can see that the largest gaps between the best and worst performances are 1.94\% (MRR) and $0.56 \%$ (Recall) on HAmazon, $0.78 \%$ (MRR) and $1.21 \%$ (Recall) on HVIDEO. This is because even if $K=1,2$, our models still consider the information of all members except that some members are modeled as a single latent user role.

\section{CONCLUSION AND Future WORK}

We have studied the task of shared account cross-domain sequential recommendation (SAC-SR) and proposed an extension to our previous work [52]. We have generalized over the previous proposal ( $\pi$-Net) with a more general framework that allows us to come up with a better performing model. Under this framework, we have reformulated $\pi$-Net as PSJNet-I and proposed a new instantiation, PSJNet-II, with different splitjoin schemes. Experimental results demonstrate that PSJNet outperforms state-of-the-art methods in terms of MRR and Recall. We have also conducted extensive analysis experiments to show the effectiveness of the two PSJNet variants.

A limitation of PSJNet is that it works better only when we have shared information in two domains that are complementary to each other. Otherwise, PSJNet only achieves com- 
TABLE VI: Analysis of the hyperparameter $K$ on the HAmazon dataset.

\begin{tabular}{|c|c|c|c|c|c|c|c|c|c|c|c|c|}
\hline \multirow{3}{*}{$K$ values } & \multicolumn{6}{|c|}{ M-domain recommendation } & \multicolumn{6}{|c|}{ B-domain recommendation } \\
\hline & \multicolumn{3}{|c|}{ MRR } & \multicolumn{3}{|c|}{ Recall } & \multicolumn{3}{|c|}{ MRR } & \multicolumn{3}{|c|}{ Recall } \\
\hline & @5 & @ 10 & @20 & @5 & @ 10 & @20 & $@ 5$ & @ 10 & @20 & @5 & @ 10 & @20 \\
\hline \multicolumn{13}{|c|}{ PSJNet-I } \\
\hline 1 & 82.45 & 82.52 & 82.54 & 84.23 & 84.69 & 85.07 & 84.72 & 84.73 & 84.73 & 85.29 & 85.35 & 85.38 \\
\hline 2 & 83.35 & 83.40 & 83.41 & 84.66 & 85.02 & 85.18 & 84.74 & 84.75 & 84.75 & 85.30 & 85.25 & 85.37 \\
\hline 3 & 83.65 & 83.68 & 83.70 & 84.81 & 85.08 & 85.30 & 84.89 & 84.89 & 84.89 & 85.32 & 85.35 & 85.38 \\
\hline 4 & 83.91 & 83.94 & 83.95 & 84.91 & 85.13 & 85.33 & 84.93 & 84.93 & 84.93 & 85.33 & 85.40 & 85.38 \\
\hline 5 & 83.73 & 83.76 & 83.78 & 84.82 & 85.08 & 85.32 & 84.94 & 84.94 & 84.94 & 85.33 & 85.38 & 85.39 \\
\hline \multicolumn{13}{|c|}{ PSJNet-II } \\
\hline 1 & 84.33 & 84.36 & 84.37 & 85.01 & 85.19 & 85.32 & 85.09 & 85.10 & 85.10 & 85.32 & 85.36 & 85.39 \\
\hline 2 & 84.08 & 84.12 & 84.13 & 84.92 & 85.15 & 85.30 & 85.13 & 85.13 & 85.13 & 85.33 & 85.36 & 85.40 \\
\hline 3 & 84.03 & 84.06 & 84.07 & 84.92 & 85.12 & 85.29 & 85.16 & 85.16 & 85.16 & 85.33 & 85.35 & 85.37 \\
\hline 4 & 84.01 & 84.04 & 84.05 & 84.88 & 85.10 & 85.28 & 85.10 & 85.10 & 85.11 & 85.32 & 85.37 & 85.38 \\
\hline 5 & 82.34 & 82.42 & 82.44 & 84.06 & 84.63 & 84.99 & 84.67 & 84.68 & 84.69 & 85.23 & 85.30 & 85.37 \\
\hline
\end{tabular}

TABLE VII: Analysis of the hyperparameter $K$ on the HVIDEO dataset.

\begin{tabular}{|c|c|c|c|c|c|c|c|c|c|c|c|c|}
\hline \multirow{3}{*}{$K$ values } & \multicolumn{6}{|c|}{ V-domain recommendation } & \multicolumn{6}{|c|}{ E-domain recommendation } \\
\hline & \multicolumn{3}{|c|}{ MRR } & \multicolumn{3}{|c|}{ Recall } & \multicolumn{3}{|c|}{ MRR } & \multicolumn{3}{|c|}{ Recall } \\
\hline & @5 & @ 10 & $@ 20$ & @5 & @ 10 & @ 20 & @5 & @ 10 & @ 20 & $@ 5$ & @ 10 & @ 20 \\
\hline \multicolumn{13}{|c|}{ PSJNet-I } \\
\hline 1 & 80.19 & 80.50 & 80.66 & 82.85 & 85.15 & 87.40 & 13.92 & 15.06 & 16.10 & 19.76 & 28.74 & 43.98 \\
\hline 2 & 80.48 & 80.75 & 80.91 & 83.08 & 85.06 & 87.31 & 14.29 & 15.47 & 16.54 & 19.83 & 28.96 & 44.77 \\
\hline 3 & 80.53 & 80.79 & 80.93 & 83.34 & 85.31 & 87.31 & 14.45 & 15.54 & 16.64 & 20.23 & 28.61 & 44.64 \\
\hline 4 & 80.51 & 80.80 & 80.95 & 83.22 & 85.34 & 87.48 & 14.63 & 15.83 & 16.88 & 20.41 & 29.61 & 45.19 \\
\hline 5 & 80.60 & 80.86 & 81.02 & 83.25 & 85.19 & 87.47 & 14.59 & 15.71 & 16.75 & 20.42 & 28.97 & 44.38 \\
\hline \multicolumn{13}{|c|}{ PSJNet-II } \\
\hline 1 & 81.93 & 82.18 & 82.32 & 84.33 & 86.17 & 88.21 & 16.17 & 17.18 & 18.13 & 21.42 & 29.23 & 43.29 \\
\hline 2 & 81.80 & 82.04 & 82.17 & 84.26 & 86.05 & 87.90 & 16.62 & 17.67 & 18.55 & 21.60 & 29.60 & 42.63 \\
\hline 3 & 81.86 & 82.08 & 82.20 & 84.14 & 85.80 & 87.53 & 16.90 & 17.94 & 18.77 & 22.42 & 30.36 & 42.51 \\
\hline 4 & 81.97 & 82.20 & 82.32 & 84.32 & 86.11 & 87.75 & 16.63 & 17.62 & 18.46 & 22.12 & 29.64 & 42.20 \\
\hline 5 & 81.78 & 82.02 & 82.14 & 83.99 & 85.67 & 87.68 & 16.78 & 17.84 & 18.66 & 22.01 & 30.07 & 42.13 \\
\hline
\end{tabular}

parable performance with state-of-the-art methods for shared account and/or cross-domain recommendations.

As to future work, PSJNet can be advanced in several directions. First, we assume the same number of latent user roles under each account in this study. This can be further improved by automatically detecting the number of user roles, e.g., adaptively setting the number of family members in smart TV scenarios. Second, we have focused on the architecture of PSJNet and have not explored alternative choices for some of its main ingredients (e.g., encoders, decoders and loss functions). It would be interesting to see whether alternative choices will further improve the performance of PSJNet. Third, side information (e.g., movie categories, attributes or labels, etc.) has been proven effective in improving recommendation performance in traditional recommendation [24, 72] and SR [11]. We hope to explore how to better incorporate side information into PSJNet for SAC-SR. Fourth, explainability is seen as important challenge for deep learning at present. Explainability is not the focus of this work, it is interesting to see how effective explanations can be produced for different stakeholders in the complex domain of shared account crossdomain sequential recommendation (SAC-SR) [51].

\section{ACKNOWLEDGEMENTS}

This work is supported by the Hybrid Intelligence Center, a 10 -year programme funded by the Dutch Ministry of Educa- tion, Culture and Science through the Netherlands Organisation for Scientific Research, https://hybrid-intelligence-centre. nl, the Natural Science Foundation of China (61672324, 61672322, 61972234, 61902219), the Natural Science Foundation of Shandong province (2016ZRE27468), the Tencent AI Lab Rhino-Bird Focused Research Program (JR201932), and the Fundamental Research Funds of Shandong University. All content represents the opinion of the authors, which is not necessarily shared or endorsed by their respective employers and/or sponsors.

\section{CODE AND DATA}

The code used to run the experiments in this paper is available at https://bitbucket.org/Catherine_Ma/sequentialrec/src/ master/tois-PsiNet/code/. The datasets released in this paper are shared at https://bitbucket.org/Catherine_Ma/sequentialrec/ src/master/tois-PsiNet/datasets/

\section{REFERENCES}

[1] Fabian Abel, Eelco Herder, Geert-Jan Houben, Nicola Henze, and Daniel Krause. Cross-system user modeling and personalization on the social web. User Modeling and User-Adapted Interaction, 23(2):169-209, 2013.

[2] Michal Aharon, Eshcar Hillel, Amit Kagian, Ronny Lempel, Hayim Makabee, and Raz Nissim. Watch-it-next: A contextual tv recommendation system. In Machine 
Learning and Knowledge Discovery in Databases, pages 180-195, Cham, 2015. Springer International Publishing.

[3] Payal Bajaj and Sumit Shekhar. Experience individualization on online tv platforms through persona-based account decomposition. In Proceedings of the 24th ACM International Conference on Multimedia, MM '16, pages 252-256, New York, NY, USA, 2016. ACM.

[4] Shlomo Berkovsky, Tsvi Kuflik, and Francesco Ricci. Cross-domain mediation in collaborative filtering. In Proceedings of the 11th International Conference on User Modeling, UM '07, pages 355-359, Berlin, Heidelberg, 2007. Springer-Verlag.

[5] Shlomo Berkovsky, Tsvi Kuflik, and Francesco Ricci. Mediation of user models for enhanced personalization in recommender systems. User Modeling and User-Adapted Interaction, 18(3):245-286, 2008.

[6] Veronika Bogina and Tsvi Kuflik. Incorporating dwell time in session-based recommendations with recurrent neural networks. In Proceedings of RecTemp Workshop co-located with ACM RecSys, RecTemp '17, pages 5759, New York, NY, USA, 2017. ACM.

[7] Bin Cao, Nathan Nan Liu, and Qiang Yang. Transfer learning for collective link prediction in multiple heterogenous domains. In Proceedings of the 27th International Conference on International Conference on Machine Learning, ICML'10, pages 159-166, USA, 2010. Omnipress.

[8] Chao Chen, Dongshen Li, Junchi Yan, and Xiaokang Yang. Modeling dynamic user preference via dictionary learning for sequential recommendation. IEEE Transactions on Knowledge and Data Engineering, pages 1-1, 2021.

[9] Leihui Chen, Jianbing Zheng, Ming Gao, Aoying Zhou, Wei Zeng, and Hui Chen. Tlrec: Transfer learning for cross-domain recommendation. In 2017 IEEE International Conference on Big Knowledge, ICBK '17, pages 167-172, New York, NY, USA, 2017. IEEE.

[10] Shuo Chen, Josh L. Moore, Douglas Turnbull, and Thorsten Joachims. Playlist prediction via metric embedding. In Proceedings of the 18th ACM SIGKDD International Conference on Knowledge Discovery and Data Mining, KDD '12, pages 714-722, New York, NY, USA, 2012. ACM.

[11] Xu Chen, Hongteng Xu, Yongfeng Zhang, Jiaxi Tang, Yixin Cao, Zheng Qin, and Hongyuan Zha. Sequential recommendation with user memory networks. In Proceedings of the Eleventh ACM International Conference on Web Search and Data Mining, WSDM '18, pages 108-116, New York, NY, USA, 2018. ACM.

[12] Zhiyong Cheng, Jialie Shen, Lei Zhu, Mohan S Kankanhalli, and Liqiang Nie. Exploiting music play sequence for music recommendation. In Proceedings of the 26th International Joint Conference on Artificial Intelligence, IJCAI '17, pages 3654-3660. AAAI Press, 2017.

[13] Zhiyong Cheng, Ying Ding, Lei Zhu, and Mohan Kankanhalli. Aspect-aware latent factor model: Rating prediction with ratings and reviews. In Proceedings of the 2018 World Wide Web Conference, WWW'18, pages
639-648, Republic and Canton of Geneva, Switzerland, 2018. International World Wide Web Conferences Steering Committee.

[14] Paolo Cremonesi and Massimo Quadrana. Cross-domain recommendations without overlapping data: Myth or reality? In Proceedings of the 8th ACM Conference on Recommender Systems, RecSys '14, pages 297-300, New York, NY, USA, 2014. ACM.

[15] Qiang Cui, Shu Wu, Qiang Liu, Wen Zhong, and Liang Wang. MV-RNN: A multi-view recurrent neural network for sequential recommendation. IEEE Transactions on Knowledge and Data Engineering, 32(2):317-331, 2020.

[16] Quan Do, Wei Liu, Jin Fan, and Dacheng Tao. Unveiling hidden implicit similarities for cross-domain recommendation. IEEE Transactions on Knowledge and Data Engineering, 33(1):302-315, 2021.

[17] Thanh-Nam Doan and Shaghayegh Sahebi. TransCrossCF: Transition-based cross-domain collaborative filtering. In 2020 19th IEEE International Conference on Machine Learning and Applications (ICMLA), pages 320-327, 2020.

[18] Tim Donkers, Benedikt Loepp, and Jürgen Ziegler. Sequential user-based recurrent neural network recommendations. In Proceedings of the Eleventh ACM Conference on Recommender Systems, RecSys '17, pages 152-160, New York, NY, USA, 2017. ACM.

[19] Ali Mamdouh Elkahky, Yang Song, and Xiaodong He. A multi-view deep learning approach for cross domain user modeling in recommendation systems. In Proceedings of the 24th International Conference on World Wide Web, WWW'15, pages 278-288, Republic and Canton of Geneva, Switzerland, 2015. International World Wide Web Conferences Steering Committee.

[20] Hui Fang, Danning Zhang, Yiheng Shu, and Guibing Guo. Deep learning for sequential recommendation: Algorithms, influential factors, and evaluations. ACM Trans. Inf. Syst., 39(1), November 2020.

[21] Aleksandr Farseev, Ivan Samborskii, Andrey Filchenkov, and Tat-Seng Chua. Cross-domain recommendation via clustering on multi-layer graphs. In Proceedings of the 40th International ACM SIGIR Conference on Research and Development in Information Retrieval, SIGIR '17, pages 195-204, New York, NY, USA, 2017. ACM.

[22] Ignacio Fernández-Tobías, Iván Cantador, Marius Kaminskas, and Francesco Ricci. Cross-domain recommender systems: A survey of the state of the art. In Proceedings of the 2nd Spanish Conference on Information Retrieval, CERI '12, pages -, 2012.

[23] Ignacio Fernández-Tobías, Paolo Tomeo, Iván Cantador, Tommaso Di Noia, and Eugenio Di Sciascio. Accuracy and diversity in cross-domain recommendations for coldstart users with positive-only feedback. In Proceedings of the 10th ACM Conference on Recommender Systems, RecSys '16, pages 119-122, New York, NY, USA, 2016. ACM.

[24] Rana Forsati, Mehrdad Mahdavi, Mehrnoush Shamsfard, and Mohamed Sarwat. Matrix factorization with explicit trust and distrust side information for improved social 
recommendation. ACM Transactions on Information Systems, 32(4):17:1-17:38, 2014.

[25] Xavier Glorot and Yoshua Bengio. Understanding the difficulty of training deep feedforward neural networks. In In Proceedings of the International Conference on Artificial Intelligence and Statistics Society for Artificial Intelligence and Statistics, AISTATS '10, pages 249-256, 2010.

[26] Ruining $\mathrm{He}$ and Julian McAuley. Ups and downs: Modeling the visual evolution of fashion trends with oneclass collaborative filtering. In Proceedings of the 25th International Conference on World Wide Web, WWW '16, pages 507-517, Republic and Canton of Geneva, Switzerland, 2016. International World Wide Web Conferences Steering Committee.

[27] Ruining He and Julian McAuley. Fusing similarity models with markov chains for sparse sequential recommendation. In Proceedings of the IEEE 16th International Conference on Data Mining, ICDM '01, pages 191-200, New York, NY, USA, 2016. IEEE.

[28] Xiangnan He, Lizi Liao, Hanwang Zhang, Liqiang Nie, $\mathrm{Xia} \mathrm{Hu}$, and Tat-Seng Chua. Neural collaborative filtering. In Proceedings of the 26th International Conference on World Wide Web, WWW '17, pages 173182, Republic and Canton of Geneva, Switzerland, 2017. International World Wide Web Conferences Steering Committee.

[29] Xiangnan He, Zhankui He, Xiaoyu Du, and Tat-Seng Chua. Adversarial personalized ranking for recommendation. In The 41st International ACM SIGIR Conference on Research and Development in Information Retrieval, SIGIR '18, pages 355-364, New York, NY, USA, 2018. ACM.

[30] Balázs Hidasi, Alexandros Karatzoglou, Linas Baltrunas, and Domonkos Tikk. Session-based recommendations with recurrent neural networks. In International Conference on Learning Representations, ICLR '16, 2016.

[31] Balázs Hidasi, Massimo Quadrana, Alexandros Karatzoglou, and Domonkos Tikk. Parallel recurrent neural network architectures for feature-rich session-based recommendations. In Proceedings of the 10th ACM Conference on Recommender Systems, RecSys '16, pages 241-248, New York, NY, USA, 2016. ACM.

[32] Guangneng $\mathrm{Hu}$, Yu Zhang, and Qiang Yang. Conet: Collaborative cross networks for cross-domain recommendation. In Proceedings of the 27th ACM International Conference on Information and Knowledge Management, CIKM '18, pages 667-676, New York, NY, USA, 2018. ACM.

[33] Liang $\mathrm{Hu}$, Jian Cao, Guandong Xu, Longbing Cao, Zhiping Gu, and Can Zhu. Personalized recommendation via cross-domain triadic factorization. In Proceedings of the 22Nd International Conference on World Wide Web, WWW '13, pages 595-606, New York, NY, USA, 2013. ACM.

[34] Jin Huang, Wayne Xin Zhao, Hongjian Dou, Ji-Rong Wen, and Edward Y. Chang. Improving sequential recommendation with knowledge-enhanced memory net- works. In The 41st International ACM SIGIR Conference on Research and Development in Information Retrieval, SIGIR '18, pages 505-514, New York, NY, USA, 2018. ACM.

[35] Jin Huang, Zhaochun Ren, Wayne Xin Zhao, Gaole He, Ji-Rong Wen, and Daxiang Dong. Taxonomy-aware multi-hop reasoning networks for sequential recommendation. In Proceedings of the Twelfth ACM International Conference on Web Search and Data Mining, WSDM '19, pages 573-581, New York, NY, USA, 2019. ACM.

[36] Po-Sen Huang, Xiaodong He, Jianfeng Gao, Li Deng, Alex Acero, and Larry Heck. Learning deep structured semantic models for web search using clickthrough data. In Proceedings of the 22Nd ACM International Conference on Information \& Knowledge Management, CIKM '13, pages 2333-2338, New York, NY, USA, 2013. ACM.

[37] Dietmar Jannach and Malte Ludewig. When recurrent neural networks meet the neighborhood for session-based recommendation. In Proceedings of the 11th ACM Conference on Recommender Systems, RecSys '17, pages 306-310, New York, NY, USA, 2017. ACM.

[38] Jyun-Yu Jiang, Cheng-Te Li, Yian Chen, and Wei Wang. Identifying users behind shared accounts in online streaming services. In The 41st International ACM SIGIR Conference on Research \&\#38; Development in Information Retrieval, SIGIR '18, pages 65-74, New York, NY, USA, 2018. ACM.

[39] Heishiro Kanagawa, Hayato Kobayashi, Nobuyuki Shimizu, Yukihiro Tagami, and Taiji Suzuki. Crossdomain recommendation via deep domain adaptation. arXiv preprint arXiv:1803.03018, 2018.

[40] Yehuda Koren, Robert Bell, and Chris Volinsky. Matrix factorization techniques for recommender systems. Computer, 42(8):30-37, 2009.

[41] Clément Lesaege, François Schnitzler, Anne Lambert, and Jean-Ronan Vigouroux. Time-aware user identification with topic models. In 2016 IEEE 16th International Conference on Data Mining, ICDM '16, pages 9971002, New York, NY, USA, 2016. IEEE.

[42] Bin Li, Qiang Yang, and Xiangyang Xue. Can movies and books collaborate? cross-domain collaborative filtering for sparsity reduction. In Proceedings of the 17th International Joint Conference on Artificial Intelligence, IJCAI '09, pages 2052-2057. AAAI Press, 2009.

[43] Haoyang Li, Xin Wang, Ziwei Zhang, Jianxin Ma, Peng Cui, and Wenwu Zhu. Intention-aware sequential recommendation with structured intent transition. IEEE Transactions on Knowledge and Data Engineering, pages $1-1,2021$.

[44] Jing Li, Pengjie Ren, Zhumin Chen, Zhaochun Ren, Tao Lian, and Jun Ma. Neural attentive session-based recommendation. In Proceedings of the 2017 ACM on Conference on Information and Knowledge Management, CIKM '17, pages 1419-1428, New York, NY, USA, 2017. ACM.

[45] Jianxun Lian, Fuzheng Zhang, Xing Xie, and Guangzhong Sun. Cccfnet: A content-boosted 
collaborative filtering neural network for cross domain recommender systems. In Proceedings of the 26th International Conference on World Wide Web Companion, WWW '17 Companion, pages 817-818, Republic and Canton of Geneva, Switzerland, 2017. International World Wide Web Conferences Steering Committee.

[46] Greg Linden, Brent Smith, and Jeremy York. Amazon.com recommendations: Item-to-item collaborative filtering. IEEE Internet Computing, 7(1):76-80, 2003.

[47] Qiang Liu, Shu Wu, Diyi Wang, Zhaokang Li, and Liang Wang. Context-aware sequential recommendation. In Proceedings of the IEEE 16th International Conference on Data Mining, ICDM '16, pages 1053-1058, New York, NY, USA, 2016. IEEE.

[48] Zhen Liu, Jingyu Tian, Lingxi Zhao, and Yanling Zhang. Attentive-feature transfer based on mapping for crossdomain recommendation. In 2020 International Conference on Data Mining Workshops (ICDMW), pages 151158, 2020.

[49] Babak Loni, Yue Shi, Martha Larson, and Alan Hanjalic. Cross-domain collaborative filtering with factorization machines. In Proceedings of the 4th Spanish Conference on Information Retrieval, CERI '14, pages 656-661, 2014.

[50] Zhongqi Lu, Erheng Zhong, Lili Zhao, Evan Wei Xiang, Weike Pan, and Qiang Yang. Selective transfer learning for cross domain recommendation. In Proceedings of the 2013 SIAM International Conference on Data Mining, SIAM '13, pages 641-649, Austin, Texas, USA, 2013.

[51] Ana Lucic, Madhulika Srikumar, Umang Bhatt, Alice Xiang, Ankur Taly, Q. Vera Liao, and Maarten de Rijke. A multistakeholder approach towards evaluating ai transparency mechanisms. In ACM CHI Workshop on Operationalizing Human-Centered Perspectives in Explainable AI. ACM, May 2021.

[52] Muyang Ma, Pengjie Ren, Yujie Lin, Zhumin Chen, Jun Ma, and Maarten de Rijke. $\pi$-net: A parallel informationsharing network for cross-domain shared-account sequential recommendations. In The 42st International ACM SIGIR Conference on Research and Development in Information Retrieval, SIGIR '19, pages 685-694, New York, NY, USA, 2019. ACM.

[53] Renfeng Ma, Qi Zhang, Jiawen Wang, Lizhen Cui, and Xuanjing Huang. Mention recommendation for multimodal microblog with cross-attention memory network. In The 41st International ACM SIGIR Conference on Research and Development in Information Retrieval, SIGIR '18, pages 195-204, New York, NY, USA, 2018. ACM.

[54] Lei Mei, Pengjie Ren, Zhumin Chen, Liqiang Nie, Jun Ma, and Jian-Yun Nie. An attentive interaction network for context-aware recommendations. In Proceedings of the 27th ACM International Conference on Information and Knowledge Management, CIKM '18, pages 157166, New York, NY, USA, 2018. ACM.

[55] Ishan Misra, Abhinav Shrivastava, Abhinav Gupta, and Martial Hebert. Cross-stitch networks for multi-task learning. In The IEEE Conference on Computer Vision and Pattern Recognition, CVPR '16, pages 3994-4003, New York, NY, USA, 2016. IEEE.

[56] Kezban Dilek Onal, Ye Zhang, Ismail Sengor Altingovde, Md Mustafizur Rahman, Pinar Karagoz, Alex Braylan, Brandon Dang, Heng-Lu Chang, Henna Kim, Quinten McNamara, Aaron Angert, Edward Banner, Vivek Khetan, Tyler McDonnell, An Thanh Nguyen, Dan Xu, Byron C. Wallace, Maarten de Rijke, and Matthew Lease. Neural information retrieval: At the end of the early years. Information Retrieval Journal, 21(2-3):111-182, June 2018.

[57] Weike Pan, Evan Wei Xiang, Nathan Nan Liu, and Qiang Yang. Transfer learning in collaborative filtering for sparsity reduction. In The 24th AAAI Conference on Artificial Intelligence, AAAI '10, pages 230-235. AAAI Press, 2010.

[58] Razvan Pascanu, Tomas Mikolov, and Yoshua Bengio. On the difficulty of training recurrent neural networks. In Proceedings of the 30th International Conference on International Conference on Machine Learning, ICML '13, pages III-1310-III-1318. JMLR.org, 2013.

[59] Massimo Quadrana, Alexandros Karatzoglou, Balzs Hidasi, and Paolo Cremonesi. Personalizing session-based recommendations with hierarchical recurrent neural networks. In Proceedings of the 11th ACM Conference on Recommender Systems, RecSys '17, pages 130-137, New York, NY, USA, 2017. ACM.

[60] Massimo Quadrana, Paolo Cremonesi, and Dietmar Jannach. Sequence-aware recommender systems. ACM Computing Surveys, 51(4):66:1-66:36, 2018.

[61] Pengjie Ren, Zhumin Chen, Jing Li, Zhaochun Ren, Jun Ma, and Maarten de Rijke. Repeatnet: A repeat aware neural recommendation machine for session-based recommendation. In The Thirty-Third AAAI Conference on Artificial Intelligence, AAAI '19, pages 4806-4813. AAAI Press, 2019.

[62] Steffen Rendle, Christoph Freudenthaler, Zeno Gantner, and Lars Schmidt-Thieme. Bpr: Bayesian personalized ranking from implicit feedback. In Proceedings of the Twenty-Fifth Conference on Uncertainty in Artificial Intelligence, UAI '09, pages 452-461, Arlington, Virginia, United States, 2009. AUAI Press.

[63] Steffen Rendle, Christoph Freudenthaler, and Lars Schmidt-Thieme. Factorizing personalized markov chains for next-basket recommendation. In Proceedings of the 19th International Conference on World Wide Web, WWW'10, pages 811-820, New York, NY, USA, 2010. ACM.

[64] Badrul Sarwar, George Karypis, Joseph Konstan, and John Riedl. Item-based collaborative filtering recommendation algorithms. In Proceedings of the 10th International Conference on World Wide Web, WWW '01, pages 285-295, New York, NY, USA, 2001. ACM.

[65] Guy Shani, David Heckerman, and Ronen I. Brafman. An mdp-based recommender system. Journal of Machine Learning Research, 6:1265-1295, 2005.

[66] Bracha Shapira, Lior Rokach, and Shirley Freilikhman. 
Facebook single and cross domain data for recommendation systems. User Modeling and User-Adapted Interaction, 23(2):211-247, 2013.

[67] Nitish Srivastava, Geoffrey Hinton, Alex Krizhevsky, Ilya Sutskever, and Ruslan Salakhutdinov. Dropout: A simple way to prevent neural networks from overfitting. Journal of Machine Learning Research, 15(1):1929-1958, 2014.

[68] Qiaoyu Tan, Jianwei Zhang, Ninghao Liu, Xiao Huang, Hongxia Yang, Jingren Zhou, and Xia Hu. Dynamic memory based attention network for sequential recommendation, 022021.

[69] Yong Kiam Tan, Xinxing Xu, and Yong Liu. Improved recurrent neural networks for session-based recommendations. In Proceedings of the 1st Workshop on Deep Learning for Recommender Systems, DLRS '16, pages 17-22, New York, NY, USA, 2016. ACM.

[70] Jiaxi Tang and Ke Wang. Personalized top-n sequential recommendation via convolutional sequence embedding. In Proceedings of the Eleventh ACM International Conference on Web Search and Data Mining, WSDM '18, pages 565-573, New York, NY, USA, 2018. ACM.

[71] Jie Tang, Sen Wu, Jimeng Sun, and Hang Su. Crossdomain collaboration recommendation. In Proceedings of the 18th ACM SIGKDD International Conference on Knowledge Discovery and Data Mining, KDD '12, pages 1285-1293, New York, NY, USA, 2012. ACM.

[72] Flavian Vasile, Elena Smirnova, and Alexis Conneau. Meta-prod2vec: Product embeddings using sideinformation for recommendation. In Proceedings of the 10th ACM Conference on Recommender Systems, RecSys '16, pages 225-232, New York, NY, USA, 2016. ACM.

[73] Koen Verstrepen and Bart Goethals. Top-n recommendation for shared accounts. In Proceedings of the 9th ACM Conference on Recommender Systems, RecSys '15, pages 59-66, New York, NY, USA, 2015. ACM.

[74] Meirui Wang, Pengjie Ren, Lei Mei, Zhumin Chen, Ma Jun, and Maarten de Rijke. A collaborative sessionbased recommendation approach with parallel memory modules. In The 42st International ACM SIGIR Conference on Research and Development in Information Retrieval, SIGIR '19, pages 345-354, New York, NY, USA, 2019. ACM.

[75] Pengfei Wang, Jiafeng Guo, Yanyan Lan, Jun Xu, Shengxian Wan, and Xueqi Cheng. Learning hierarchical representation model for next basket recommendation. In Proceedings of the 38th International ACM SIGIR Conference on Research and Development in Information Retrieval, SIGIR '15, pages 403-412, New York, NY, USA, 2015. ACM.

[76] Xiang Wang, Xiangnan He, Liqiang Nie, and Tat-Seng Chua. Item silk road: Recommending items from information domains to social users. In Proceedings of the 40th International ACM SIGIR Conference on Research and Development in Information Retrieval, SIGIR '17, pages 185-194, New York, NY, USA, 2017. ACM.

[77] Zhijin Wang, Yan Yang, Liang He, and Junzhong Gu. User identification within a shared account: Improving ip-tv recommender performance. In Advances in
Databases and Information Systems, ADBIS '14, pages 219-233, Cham, 2014. Springer International Publishing.

[78] Xiang Wu, Qi Liu, Enhong Chen, Liang He, Jingsong Lv, Can Cao, and Guoping Hu. Personalized next-song recommendation in online karaokes. In Proceedings of the 7th ACM Conference on Recommender Systems, RecSys '13, pages 137-140, New York, NY, USA, 2013. ACM.

[79] Yan Yan, Mingkui Tan, Ivor W. Tsang, Yi Yang, Qinfeng Shi, and Chengqi Zhang. Fast and low memory cost matrix factorization: Algorithm, analysis, and case study. IEEE Transactions on Knowledge and Data Engineering, 32(2):288-301, 2020.

[80] Shuo Yang, Somdeb Sarkhel, Saayan Mitra, and Viswanathan Swaminathan. Personalized video recommendations for shared accounts. In 2017 IEEE International Symposium on Multimedia, ISM '17, pages 256259, 2017.

[81] Yan Yang, Qinmin Hu, Liang He, Minjie Ni, and Zhijin Wang. Adaptive temporal model for iptv recommendation. In Web-Age Information Management, WAIM '15, pages 260-271, Cham, 2015. Springer International Publishing.

[82] Ghim-Eng Yap, Xiao-Li Li, and S Yu Philip. Effective next-items recommendation via personalized sequential pattern mining. In Database Systems for Advanced Applications, DASFAA '12, pages 48-64, Berlin, Heidelberg, 2012. Springer Berlin Heidelberg.

[83] Feng Yu, Qiang Liu, Shu Wu, Liang Wang, and Tieniu Tan. A dynamic recurrent model for next basket recommendation. In Proceedings of the 39th International ACM SIGIR Conference on Research and Development in Information Retrieval, SIGIR '16, pages 729-732, New York, NY, USA, 2016. ACM.

[84] Amy Zhang, Nadia Fawaz, Stratis Ioannidis, and Andrea Montanari. Guess who rated this movie: Identifying users through subspace clustering. In Proceedings of the Twenty-Eighth Conference on Uncertainty in Artificial Intelligence, UAI '12, pages 944-953, Arlington, Virginia, United States, 2012. AUAI Press.

[85] Hongwei Zhang, Xiangwei Kong, and Yujia Zhang. Selective knowledge transfer for cross-domain collaborative recommendation. IEEE Access, pages 1-1, 2021.

[86] Yafeng Zhao, Jian Cao, and Yudong Tan. Passenger prediction in shared accounts for flight service recommendation. In The 10th International Conference on Asia-Pacific Services Computing, APSCC '16, pages 159-172, Cham, 2016. Springer International Publishing.

[87] Fuzhen Zhuang, Ping Luo, Hui Xiong, Yuhong Xiong, Qing He, and Zhongzhi Shi. Cross-domain learning from multiple sources: A consensus regularization perspective. Transactions on Knowledge and Data Engineering, 22: 1664-1678, 2010.

[88] Fuzhen Zhuang, Yingmin Zhou, Fuzheng Zhang, Xiang Ao, Xing Xie, and Qing He. Sequential transfer learning: Cross-domain novelty seeking trait mining for recommendation. In Proceedings of the 26th International Conference on World Wide Web Companion, WWW'17 
Companion, pages 881-882, Republic and Canton of Geneva, Switzerland, 2017. International World Wide Web Conferences Steering Committee.

[89] Andrew Zimdars, David Maxwell Chickering, and Christopher Meek. Using temporal data for making recommendations. In Proceedings of the Seventeenth Conference on Uncertainty in Artificial Intelligence, UAI '01, pages 580-588, San Francisco, CA, USA, 2001. Morgan Kaufmann Publishers Inc. 